\title{
Comparison of dynamic headspace methods used for the analysis of the volatile composition of Spanish PDO ewe's raw milk cheeses
}

\author{
Luis Javier R. BARRONa*, Yolanda REDONDO ${ }^{a}$, María ORTIGOSA ${ }^{\mathrm{d}}$, \\ María CARBONELL ${ }^{c}$, Susana GARCÍA ${ }^{\mathrm{d}}$, Paloma TORRE ${ }^{\mathrm{d}}$, \\ Mertxe DE RENOBALEs ${ }^{b^{*}}$, Estrella FERNÁNDEZ-GARCÍA ${ }^{c^{*}}$ \\ a Tecnología de los Alimentos, Universidad del País Vasco/Euskal Herriko Unibertsitatea, \\ Paseo de la Universidad 7, 01006 Vitoria-Gasteiz, Spain \\ b Bioquímica y Biología Molecular, Facultad de Farmacia, Universidad del País Vasco/Euskal Herriko \\ Unibertsitatea, Paseo de la Universidad 7, 01006 Vitoria-Gasteiz, Spain \\ c Tecnología de Alimentos. Instituto Nacional de Investigación y Tecnología Agraria y Alimentaria, \\ Carretera de La Coruña, Km 7, 28040, Madrid, Spain \\ d Nutrición y Bromatología, Universidad Pública de Navarra, Campus Arrosadía, 31006 Pamplona, Spain
}

Received 15 October 2004 - Accepted 17 May 2005

Published online 1 October 2005

\begin{abstract}
Different dynamic headspace (DHS) methods used for determination of volatile compounds in cheeses were compared. Three different laboratories using gas chromatography coupled to mass spectrometry carried out the analyses of volatile compounds of Spanish Protected Designation of Origin ewe's raw milk cheeses. One laboratory used "purge and trap" (P\&T) with thermal desorption of the trap by ultrafast microwave heating (MWH) and the other two laboratories used P\&T with thermal desorption of the trap by resistive heating $(\mathrm{RH})$. Each method detected around 78-85 different individual compounds which belonged to different chemical groups, namely, acids, alcohols, ketones, esters, aldehydes, sulphur compounds, hydrocarbons and terpenes. Using the P\&T-MHW method, larger percentages of acids and aldehydes in the cheeses were found compared with the P\&T-RH methods, whereas the contrary occurred for the percentages of alcohols and ketones. Differences in the percentages of some volatile compounds such as ketones, alcohols and acids between the laboratories using P\&T-RH under different analytical conditions were also recorded. The results pointed out differences in the selectivity for the extraction of various volatile compounds from cheese samples according to the DHS methods used, particularly when using tenax or graphitised carbon as trap absorbents. In spite of these differences, both P\&T-MWH and P\&T$\mathrm{RH}$ methods were equally satisfactory for the comparison of the different cheese varieties. However, each of the DHS methods was more suitable for the characterisation of the volatile compounds of a particular cheese type.
\end{abstract}

cheese / volatile compound / dynamic headspace analysis / inter-laboratory study

摘要 - 动态顶空法分析西班牙原产地名号保护的羊奶干酪中挥发性化合物的对比研究。本 文比较了不同的动态顶空法测定干酪中挥发性化合物。分别在 3 个不同的实验室, 采用气 一质联机方法分析了西班牙原产地名号保护 (PDO)、原料奶未经巴氏杀菌的羊奶干酪中挥 发性化合物。其中一个实验室采用“吹扫 - 捕集” (P\&T) 方法捕集挥发性气体, 然后用超

* Corresponding authors ( 通讯作者):

luisjavier.rbarron@ehu.es; paloma@unavarra.es; mertxe.derenobales@ehu.es; fgarcia@inia.es 
速微波加热 (MWH) 的方法解析气体，另外两个实验室也采用 “吹扫 - 捕集” 方法捕集挥发 性气体, 但用热电阻加热 $(\mathrm{RH})$ 方法解析气体。每种方法都能检测出 78-85 种不同的挥发性 化合物, 这些化合物分属于酸、醇、酮、酯、醛、含硫化合物、烃类、萜烯化合物。两种 方法测得结果的比较分析表明, 采用吹扫 - 捕集 - 微波加热方法测定的挥发性化合物中酸和 醛占的比例较大, 而采用吹扫 - 捕集 - 热电阻加热方法所测定挥发性化合物中醇和酮所占的 比例较大。同时比较了均使用吹扫 - 捕集 - 热电阻加热方法的两个实验室的分析结果, 由于 分析条件的不同, 测定结果中一些挥发性化合物, 如酮、醇和酸占总挥发性化合物的百分 比有一定的差异。试验结果表明由于不同的动态顶空法对干酪样品中挥发性化合物提取物 的选择性不同, 使得分析结果存在一定的差异, 特别是使用 tenax 和石墨作吸附剂时, 这种 差异非常显著。尽管两种方法的测定结果之间存在一定的差异，经对不同种类干酪的测定 结果进行比较和分析, 测定结果还是非常满意的。然而每种动态顶空法都有其适合于特定 类型干酪中挥发性化合物的分析。

\section{干酪 / 挥发性化合物 / 动态顶空分析 / 实验室研究}

Résumé - Comparaison des méthodes d'extraction par espace de tête dynamique employées pour l'analyse des composés volatils des fromages espagnols d'Appellation d'Origine Protégée produits à partir de lait cru de brebis. Différentes méthodes d'extraction par espace de tête dynamique pour l'analyse des composés volatils du fromage ont été comparées. Trois laboratoires utilisant la chromatographie en phase gazeuse couplée à la spectrométrie de masse ont analysé cinq variétés de fromages espagnols d'appellation d'origine protégée fabriqués à partir de lait cru de brebis. Un des laboratoires a employé un système de « purge and trap » (P\&T) avec désorption thermique du piège par chauffage ultra rapide par micro-ondes (CMO) et les autres un système de P\&T avec désorption thermique du piège par chauffage résistif (CR). Chaque méthode a permis de détecter environ 78-85 composés individuels différents, qui appartenaient à différents groupes chimiques, tels que acides, alcools, cétones, esters, aldéhydes, composés soufrés, hydrocarbures et terpènes. Des pourcentages plus élevés d'acides et d'aldéhydes, et moins élevés d'alcools et de cétones, ont été trouvés en utilisant la méthode P\&T-CMO comparativement aux méthodes P\&T-CR. De plus, des différences dans les pourcentages de certains composés volatils, tels que cétones, alcools et acides ont également été observées entre les laboratoires utilisant le système de P\&T-CR dans des conditions différentes. Les résultats soulignent des différences en termes de sélectivité d'extraction des volatils à partir des échantillons de fromage selon la technique d'extraction employée, et en particulier selon la nature de l'absorbant du piège (tenax ou charbon). Malgré ces différences, les deux méthodes P\&T-CMO et P\&T-CR se sont révélées également satisfaisantes pour comparer différentes variétés de fromages. Cependant, chacune des méthodes d'extraction était plus appropriée pour caractériser les composés volatils pour un type de fromage en particulier.

\section{fromage / composé volatil / espace de tête dynamique / étude inter-laboratoire}

\section{INTRODUCTION}

The unique flavour of Protected Designation of Origin (PDO) cheeses is most important for the successful commercialisation of these products. One of the principal aims of the PDO Regulatory Boards is to characterise the typical flavour of their cheeses in order to offer consumers a unique product which can be clearly differentiated from other cheese varieties. During the last few years, it has been suggested that the volatile composition and flavour sensory properties can be used to discriminate between different cheese varieties $[4,12$, $13,29]$. Cheeses may contain many volatile compounds such as acids, alcohols, carbonyl compounds, esters, lactones, furans, nitrogen-containing compounds, sulphur and phenolic compounds, hydrocarbons and terpenes, which differ quantitatively from one variety to another [11]. These compounds are often analysed by gas chromatography (GC) coupled to mass spectrometry (MS), but it is always necessary to include a prior step involving the extraction and pre-concentration of the volatile fraction [52]. Several authors have pointed out that there is no ideal method for the extraction, concentration and injection of the volatile compounds into the chromatographic column, and that the results of the analysis will be different according to the technique used [32]. One critical aspect for cheese researchers 
is to select the most suitable extraction technique which allows one to obtain a volatile profile most closely related to the sensory properties of the cheese [28]. Numerous techniques such as simultaneous distillation-extraction $[13,21]$, high-vacuum distillation [20, 38], supercritical fluid extraction [26], static headspace [14, 41], dynamic headspace (DHS) [49, 51], solidphase microextraction $[30,31]$, and direct thermal desorption [50] have been used for the extraction of flavour compounds in cheese [32]. Nowadays DHS is being frequently used $[2,17,28,47,48,51]$. In this technique, the volatile components of the gas phase are continuously removed and concentrated in a trap or absorbed onto an inert support. This headspace system is often referred to as "purge and trap" (P\&T) [39]. Thermal desorption of the trap by resistive heating $(\mathrm{RH})$ is extensively used to transfer the volatile components onto the capillary column, cryofocusing being recommended to achieve a narrow injection band $[2,47,49]$. Thermal desorption of the trap by ultrafast microwave heating (MWH) has also been used to very rapidly transfer the volatile components into the capillary column without the need for cryofocusing [1, 3, 5, 45]. P\&T-RH has been compared with other techniques, particularly with SDE, for the analysis of volatiles of cheese [8, 27, 50]. However, few studies have focused on the comparison between different DHS methods. Some authors have concluded that cheese volatile analysis carried out by different DHS methods yield different quantitative and even qualitative results [5, 49].

Idiazabal, Manchego, Roncal, Zamorano and La Serena cheeses are the Spanish PDO cheese varieties made from raw ewe's milk [19]. Only Manchego and Zamorano cheeses may also be manufactured from pasteurised milk. The first four varieties are semi-hard cheeses coagulated with animal rennet, whereas La Serena cheese is a soft cheese variety coagulated with vegetable rennet [33-37]. The main characteristics of these PDO cheeses are shown in Table I. Studies on the volatile composition of Idiazabal, Manchego, Roncal, Zamorano and La Serena cheeses are scarce. Manchego cheese is the best known among the five varieties, but few studies have described the volatile composition of cheeses made from raw ewe's milk [15, 21, 51]. Very few papers have described the volatile composition of Idiazabal [26], Zamorano [16], Roncal $[25,42]$ and La Serena cheeses $[6,7]$.

The objective of the present study was to compare the results obtained by three different laboratories using different DHS methods in the GC-MS analysis of the volatile compounds of these PDO cheeses. One laboratory used semi-automatic P\&T$\mathrm{MWH}$, and the other two laboratories used automatic P\&T-RH.

\section{MATERIALS AND METHODS}

\subsection{Cheese samples}

Two batches of each PDO cheese were produced during two consecutive weeks in April. Cheeses were manufactured according to the rules approved by the PDO Regulatory Councils for the production of Idiazabal, Manchego, Roncal, Zamorano and La Serena cheeses [33-37]. All cheeses were sampled for analyses after six months of ripening except for La Serena cheeses which were sampled after two months of ripening (Tab. I). Two cheeses per batch and per variety were taken as duplicate samples. Cheeses were cut into three sectors and one sector of each cheese was transported in portable coolers $\left(3-4{ }^{\circ} \mathrm{C}\right)$ to each of the three laboratories collaborating in this study. Cheese sectors were wrapped in aluminium foil, vacuum-packed and stored at $-40{ }^{\circ} \mathrm{C}$ until analysis. Prior to volatile analysis vacuum-packed cheese sectors were thawed overnight at $5{ }^{\circ} \mathrm{C}$. Samples from the centre of the cheese sectors were taken for volatile analyses.

\subsection{Analysis of volatile compounds}

Three laboratories which were using DHS directly coupled to GC-MS for the analysis of the volatiles of the cheeses collaborated in this study. One of the laboratories analysed the volatile compounds by semi-automatic P\&T-MWH $(\operatorname{method} \boldsymbol{A})$, and the other two laboratories used automatic P\&T-RH (methods $\boldsymbol{B}$ and $\boldsymbol{C}$ ). 


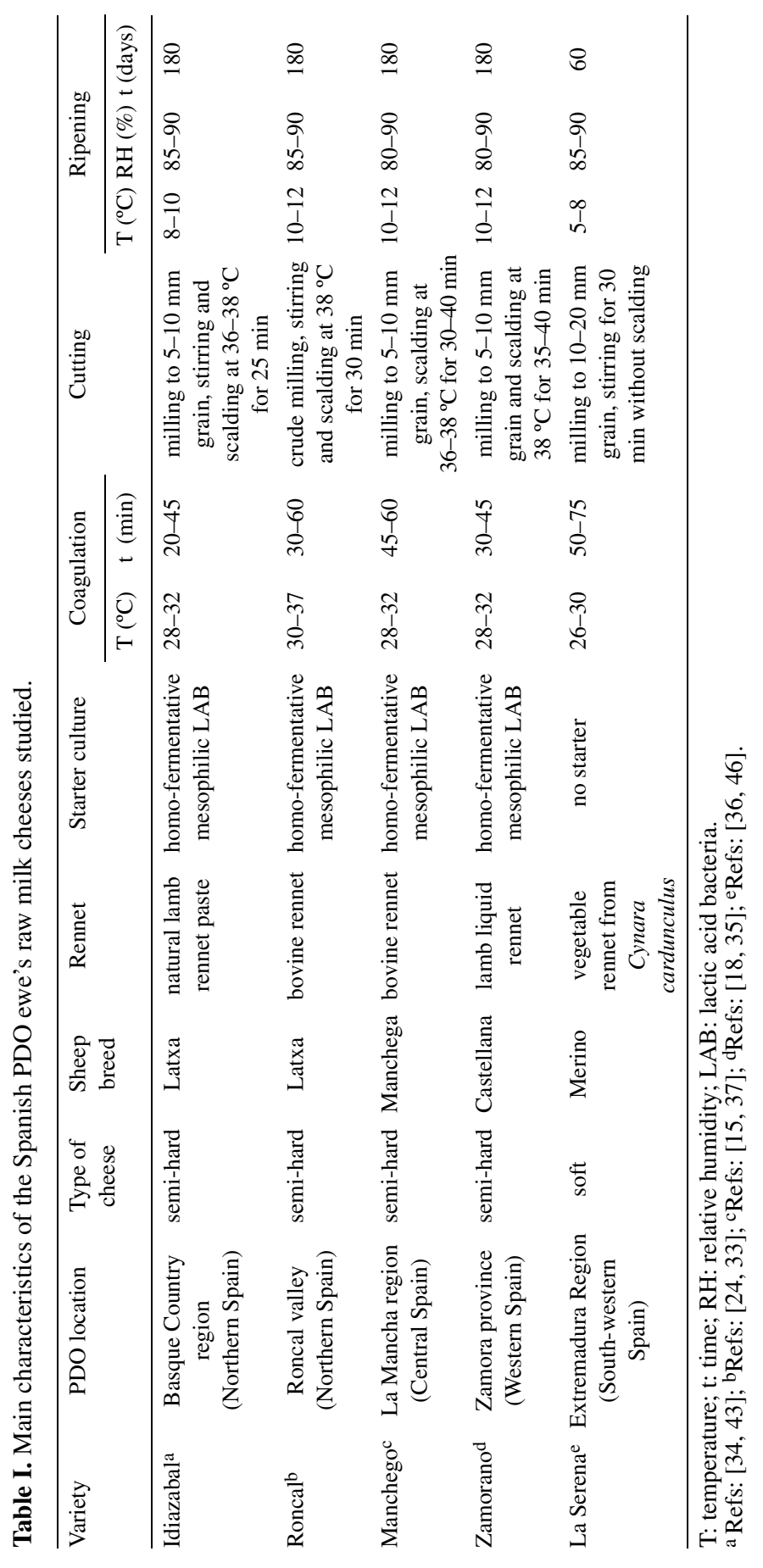




\subsubsection{Method A}

One hundred grams of cheese were homogenised in an analytical blender at 10$12^{\circ} \mathrm{C}$. An aliquot $(15 \mathrm{~g})$ of the grated cheese was placed in a $50-\mathrm{mL}$ gas washing flask. Volatile compounds were extracted with a CL-2 purge system (Chromlab, Barcelona, Spain) using ultrapure helium as purge gas and were trapped onto a graphitised carbon cartridge (1-010R, Rektorik, Meyrin, Switzerland). The DHS conditions were: $100 \mathrm{~mL} \cdot \mathrm{min}^{-1}$ purge gas flow for $30 \mathrm{~min}$, at $40{ }^{\circ} \mathrm{C}$. The volatiles concentrated in the carbon cartridge were desorbed with MW1A microwave desorption equipment (Rektorik) at 550-590 × 10-6 A.h desorption energy for $10 \mathrm{~s}$. The MW-1A desorption equipment was connected to an 8000 series GC apparatus coupled to an 800 MD MS detector (Fisons Instruments, Milan, Italy). The transfer line from the desorption equipment to the GC oven was held at $250^{\circ} \mathrm{C}$. Analyses were performed using a SUPELCOWAX ${ }^{\mathrm{TM}}$ (Supelco, Bellefonte, Palo Alto, USA) capillary column (60 m long; $0.25 \mathrm{~mm}$ ID) coated with cross-linked polyethylene glycol (0.25 $\mu \mathrm{m}$ film thickness). Oven temperature was initially held at $40^{\circ} \mathrm{C}$ for $10 \mathrm{~min}$, then increased to $240^{\circ} \mathrm{C}$ at a rate of $5^{\circ} \mathrm{C} \cdot \mathrm{min}^{-1}$, and finally held at $240^{\circ} \mathrm{C}$ for $15 \mathrm{~min}$. The carrier gas (helium) flow rate was $1.0 \mathrm{~mL} \cdot \mathrm{min}^{-1}$ and the split ratio 1:5. The transfer line from the GC apparatus to the MS detector was held at $250{ }^{\circ} \mathrm{C}$. The MS detector operated in full scan mode (total ion chromatogram at $1.85 \mathrm{scans} \cdot \mathrm{s}^{-1}$ ) from 19 to $250 \mathrm{amu}$. Ionisation was performed by electron impact at $70 \mathrm{eV}$ and the source temperature was $200{ }^{\circ} \mathrm{C}$. Peak identification was done by comparing the mass spectra with the NBS (National Bureau of Standards, USA) and NIST (National Institute of Standards and Technology, USA) libraries, and by comparison of their retention factors with authentic standards when available. The retention factor $(\mathrm{k})$ for one peak was calculated applying the formula (1):

$$
\mathrm{k}=\left(\mathrm{t}_{\mathrm{R}}-\mathrm{t}_{\mathrm{M}}\right) / \mathrm{t}_{\mathrm{M}}
$$

where $t_{R}$ and $t_{M}$ are the retention times for the peak and for an unretained peak, respectively. Quantification was carried out by the total ion current. Arbitrary units (peak area) were used.

\subsubsection{Method B}

Ten grams of cheese were homogenised in an analytical blender at $10-12^{\circ} \mathrm{C}$ with $10 \mathrm{~g}$ of $\mathrm{Na}_{2} \mathrm{SO}_{4}$ and $100 \mu \mathrm{L}$ internal standard aqueous solution prepared with $13 \mathrm{mmol} \cdot \mathrm{L}^{-1}$ borneol (Sigma-Aldrich Química), and the mixture was placed in a suitable U vial (needle sparger). Volatile compounds were extracted using ultrapure helium as purge gas in a 4460A automatic P\&T apparatus (O.I. Analytical, College Station, USA). The DHS conditions were: $40 \mathrm{~mL} \cdot \mathrm{min}^{-1}$ purge gas flow for $20 \mathrm{~min}$ at $40^{\circ} \mathrm{C}$. Volatile compounds were concentrated in a Tenax trap (O.I. Analytical) at room temperature. The transfer line and valves were kept at 120 and $180^{\circ} \mathrm{C}$, respectively. The volatiles were desorbed from the trap at $220^{\circ} \mathrm{C}$ for 1 min directly into the GC injection port. The analysis of the volatile compounds was carried out in a HP 6890 GC-MS apparatus equipped with a HP INNOWAX (Agilent Technologies, Las Rozas, Spain) capillary column (60 m long; $0.25 \mathrm{~mm}$ ID) coated with cross-linked polyethylene glycol (0.25 $\mu \mathrm{m}$ film thickness). Oven temperature was initially held at $32{ }^{\circ} \mathrm{C}$ for $7 \mathrm{~min}$, then increased to $220{ }^{\circ} \mathrm{C}$ at a rate of $6{ }^{\circ} \mathrm{C} \cdot \mathrm{min}^{-1}$, and finally held at $220^{\circ} \mathrm{C}$ for $5 \mathrm{~min}$. The carrier gas (helium) flow rate was $1.5 \mathrm{~mL} \cdot \mathrm{min}^{-1}$ for $1 \mathrm{~min}$ and then at a constant flow rate of $1 \mathrm{~mL} \cdot \mathrm{min}^{-1}$, with a split ratio of 1:30. Injection port temperature was $200{ }^{\circ} \mathrm{C}$. The transfer line from the GC apparatus to the MS detector was held at $220^{\circ} \mathrm{C}$. Detection was performed with the HP 5973 MS detector operating in full scan mode (total ion chromatogram at $3 \mathrm{scan} \cdot \mathrm{s}^{-1}$ ) from 19 to $250 \mathrm{amu}$. Ionisation was performed by electron impact at $70 \mathrm{eV}$ and the detector and source temperatures were 108 and $220{ }^{\circ} \mathrm{C}$, respectively. Peak identification was done by comparing the mass spectra with the Wiley 275 library (Wiley \& Sons Inc., Germany), and by comparison of their retention factors $(\mathrm{k})$ with authentic standards when available. Quantification was carried out by the total ion current, with reference to the borneol peak. Arbitrary units (peak area) were used. 


\subsubsection{Method C}

Fifteen grams of cheese were homogenised in an analytical blender at $10-12^{\circ} \mathrm{C}$ with $20 \mathrm{~g} \mathrm{Na}_{2} \mathrm{SO}_{4}$ and $75 \mu \mathrm{L}$ internal standard aqueous solution prepared with $0.5 \mathrm{mg} \cdot \mathrm{mL}^{-1}$ cyclohexanone (Sigma-Aldrich Química, Alcobendas, Spain). An aliquot $(2.25 \mathrm{~g})$ of the mixture was subjected to DHS using ultrapure helium as purge gas in a HP 7695 automatic P\&T apparatus (Hewlett-Packard, Palo Alto, USA). The DHS conditions were: $45 \mathrm{~mL} \cdot \mathrm{min}^{-1}$ purge gas flow for $20 \mathrm{~min}$ at $50{ }^{\circ} \mathrm{C}$, with $10 \mathrm{~min}$ of previous equilibration. Volatile compounds were concentrated in a Tenax trap (Tekmar, Cincinnati, USA) maintained at $25^{\circ} \mathrm{C}$ and 6.5 psig back pressure. The transfer line, moisture trap and valves were kept at $200{ }^{\circ} \mathrm{C}$. The trap was subjected to dry purge for $0.5 \mathrm{~min}$ and the volatile compounds were desorbed for $1 \mathrm{~min}$ at $230^{\circ} \mathrm{C}$ directly into the GC injection port. The analysis of the volatile compounds was carried out in a HP 6890 GC-MS apparatus equipped with a HP INNOWAX capillary column as in method $\boldsymbol{B}$. Oven temperature was initially held at $45^{\circ} \mathrm{C}$ for $17 \mathrm{~min}$, then increased to $110^{\circ} \mathrm{C}$ at a rate of $4^{\circ} \mathrm{C} \cdot \mathrm{min}^{-1}$, then held at $110^{\circ} \mathrm{C}$ for $10 \mathrm{~min}$, and finally increased to $240^{\circ} \mathrm{C}$ at a rate of $15^{\circ} \mathrm{C} \cdot \mathrm{min}^{-1}$. The carrier gas (helium) flow rate was $1.4 \mathrm{~mL} \cdot \mathrm{min}^{-1}$ and the split ratio $1: 20$. Injection port temperature was $220{ }^{\circ} \mathrm{C}$. The transfer line from the GC apparatus to the MS detector was held at $240^{\circ} \mathrm{C}$. Detection was performed with the HP 5973 MS detector as in method $\boldsymbol{B}$ operating in full scan mode from 33 to $200 \mathrm{amu}$, and the detector and source temperatures were 150 and $230{ }^{\circ} \mathrm{C}$, respectively. Peak identification and quantification were done as described in method $\boldsymbol{B}$ using cyclohexanone as the reference peak.

\subsection{Statistical analysis}

The SPSS statistical package, version 11.0 (SPSS Inc., Michigan, USA) was used for the statistical analysis. Analysis of variance (ANOVA) and multiple comparison tests were applied to determine the presence of significant $(P \leq 0.05)$ differences in the volatile composition of each type of PDO sample cheese analysed by the different DHS methods. Stepwise discriminant analysis was applied to classify the cheese samples according to the method used. Also, discriminant analyses were applied separately for each method to classify the cheese samples according to the PDO cheese variety. Wilk's lambda criterion was used for selecting discriminant variables [22].

\section{RESULTS AND DISCUSSION}

Table II lists the volatile compounds detected in the headspace of all the cheese samples analysed by the three laboratories using the different DHS methods $(\boldsymbol{A}, \boldsymbol{B}$ and $\boldsymbol{C}$ ). A total of 137 volatile compounds were detected by all three methods combined. These compounds belonged to different chemical groups, namely: methyl, ethyl, propyl, butyl, higher alkyl and branched alkyl esters; alkanes, aromatic and unsaturated hydrocarbons; primary, secondary, branched-chain, oxy ether and aromatic alcohols; straight, branched-chain and aromatic aldehydes; methyl, propyl and aromatic ketones; diketones; straight and branched-chain acids; sulphur compounds; and terpenes. Esters, hydrocarbons and alcohols were chemical families with a large number of different individual compounds (40, 30 and 23 compounds, respectively), whereas less than 15 compounds were found for chemical families such as aldehydes (14), ketones (14), acids (10), sulphur compounds (3) and terpenes (3) (Tab. II). Each method detected around 78-85 different individual compounds which varied with the analytical conditions used. Thus, there were 65 different compounds between methods $\boldsymbol{A}$ and $\boldsymbol{B}, 72$ different compounds between methods $\boldsymbol{A}$ and $\boldsymbol{C}$, and 55 distinct compounds between methods $\boldsymbol{B}$ and $\boldsymbol{C}$. Therefore, the volatile compositions reported by the three methods were very different (Tab. II). Only 39 of the 137 compounds were detected by each of the three methods, 33 of the 137 compounds were detected by two of the three methods, whereas the remaining 65 compounds were detected by one of the methods only (not always the same one). Esters and hydrocarbons were the volatile compounds that varied the most 
Table II. Volatile compounds found in the Spanish PDO cheeses made from ewe's raw milk using different DHS methods.

\begin{tabular}{|c|c|c|c|c|c|c|}
\hline \multirow{2}{*}{ Compounds } & \multicolumn{2}{|c|}{ Method $\boldsymbol{A}^{\mathrm{a}}$} & \multicolumn{2}{|c|}{ Method $\boldsymbol{B}^{\mathrm{b}}$} & \multicolumn{2}{|c|}{ Method $C^{b}$} \\
\hline & $\mathrm{k}$ & I & $\mathrm{k}$ & I & $\mathrm{k}$ & I \\
\hline \multicolumn{7}{|l|}{ Methyl esters } \\
\hline methyl ethanoate & & ND & 34.10 & $\mathrm{~T}$ & 38.48 & $\mathrm{P}$ \\
\hline methyl butanoate & & ND & & ND & 85.48 & $\mathrm{~T}$ \\
\hline $\begin{array}{l}\text { methyl 2-hydroxy-4-methyl- } \\
\text { pentanoate }\end{array}$ & & ND & 127.20 & $\mathrm{~T}$ & & ND \\
\hline methyl hexanoate & & ND & & ND & 150.10 & $\mathrm{P}$ \\
\hline \multicolumn{7}{|l|}{ Ethyl esters } \\
\hline ethyl ethanoate & 39.38 & $\mathrm{~T}$ & 40.95 & $\mathrm{P}$ & 49.90 & $\mathrm{P}$ \\
\hline ethenyl ethanoate & 54.14 & $\mathrm{~T}$ & & ND & & ND \\
\hline ethyl propanoate & & ND & 51.90 & $\mathrm{~T}$ & 72.90 & $\mathrm{P}$ \\
\hline ethyl 2-methylpropanoate & & ND & 54.10 & $\mathrm{~T}$ & 76.19 & $\mathrm{~T}$ \\
\hline ethyl butanoate & 63.21 & $\mathrm{P}$ & 60.15 & $\mathrm{P}$ & 105.91 & $\mathrm{P}$ \\
\hline ethyl 2-methylbutanoate & & ND & & ND & 111.33 & $\mathrm{~T}$ \\
\hline ethyl 3-methylbutanoate & & ND & 66.10 & $\mathrm{~T}$ & 116.19 & $\mathrm{~T}$ \\
\hline ethyl pentanoate & & ND & & ND & 136.62 & $\mathrm{~T}$ \\
\hline ethyl hexanoate & 92.66 & $\mathrm{P}$ & 97.00 & $\mathrm{P}$ & 160.91 & $\mathrm{P}$ \\
\hline ethyl heptanoate & 104.72 & $\mathrm{~T}$ & 109.50 & $\mathrm{P}$ & & ND \\
\hline ethyl octanoate & 116.79 & $\mathrm{P}$ & 121.50 & $\mathrm{~T}$ & 217.81 & $\mathrm{P}$ \\
\hline ethyl decanoate & & ND & & ND & 239.62 & $\mathrm{P}$ \\
\hline ethyl benzoate & & ND & & ND & 244.52 & $\mathrm{~T}$ \\
\hline \multicolumn{7}{|l|}{ Propyl esters } \\
\hline propyl ethanoate & 52.79 & $\mathrm{~T}$ & & ND & 81.00 & $\mathrm{P}$ \\
\hline propyl butanoate & 76.17 & $\mathrm{~T}$ & & ND & 133.19 & $\mathrm{P}$ \\
\hline propyl 3-methylbutanote & & ND & & ND & 141.57 & $\mathrm{~T}$ \\
\hline propyl hexanoate & & ND & 94.75 & $\mathrm{~T}$ & 183.62 & $\mathrm{~T}$ \\
\hline \multicolumn{7}{|l|}{ Butyl esters } \\
\hline butyl ethanoate & 68.66 & $\mathrm{~T}$ & 72.65 & $\mathrm{~T}$ & 118.10 & $\mathrm{P}$ \\
\hline butyl 2-methylpropanoate & & ND & & ND & 139.48 & $\mathrm{P}$ \\
\hline butyl butanoate & 90.90 & $\mathrm{~T}$ & & ND & 152.67 & $\mathrm{~T}$ \\
\hline butyl hexanoate & & ND & & ND & 213.62 & $\mathrm{~T}$ \\
\hline butyl octanoate & 116.79 & $\mathrm{~T}$ & & ND & & ND \\
\hline \multicolumn{7}{|l|}{ Higher alkyl esters } \\
\hline hexyl ethanoate & 106.14 & $\mathrm{~T}$ & & ND & & ND \\
\hline hexyl hexanoate & 122.45 & $\mathrm{~T}$ & & ND & & ND \\
\hline heptyl hexanoate & & ND & 108.50 & $\mathrm{~T}$ & & ND \\
\hline \multicolumn{7}{|l|}{ Branched alkyl esters } \\
\hline 1-methylpropyl ethanoate & 55.07 & $\mathrm{~T}$ & 57.85 & $\mathrm{~T}$ & 85.76 & $\mathrm{P}$ \\
\hline 2-methylpropyl ethanoate & & ND & & ND & 97.10 & $\mathrm{~T}$ \\
\hline
\end{tabular}


Table II. Continued.

\begin{tabular}{|c|c|c|c|c|c|c|}
\hline \multirow{2}{*}{ Compounds } & \multicolumn{2}{|c|}{ Method $\boldsymbol{A}^{\mathrm{a}}$} & \multicolumn{2}{|c|}{ Method $\boldsymbol{B}^{\mathrm{b}}$} & \multicolumn{2}{|c|}{ Method $\boldsymbol{C}^{\mathrm{b}}$} \\
\hline & $\mathrm{k}$ & I & $\mathrm{k}$ & I & $\mathrm{k}$ & I \\
\hline 3-methylbutyl ethanoate & 76.66 & $\mathrm{~T}$ & 75.15 & $\mathrm{~T}$ & 133.19 & $P$ \\
\hline $\begin{array}{l}\text { 2-methylpropyl 2-methyl- } \\
\text { propanoate }\end{array}$ & & ND & 81.50 & $\mathrm{~T}$ & & ND \\
\hline 1-methylpropyl butanoate & & ND & & ND & 134.81 & $\mathrm{~T}$ \\
\hline 2-methylpropyl butanoate & 77.14 & $\mathrm{~T}$ & & ND & 142.43 & $\mathrm{~T}$ \\
\hline 3-methylbutyl butanoate & & ND & 94.90 & $\mathrm{~T}$ & & ND \\
\hline 1-methylpropyl hexanoate & & ND & & ND & 183.86 & $\mathrm{~T}$ \\
\hline 2-methylpropyl hexanoate & 103.86 & $\mathrm{~T}$ & 108.65 & $\mathrm{~T}$ & 193.76 & $\mathrm{~T}$ \\
\hline 2-butoxyethyl ethanoate & 119.52 & $\mathrm{~T}$ & & ND & & ND \\
\hline 3-methylbutyl hexanoate & & ND & & ND & 221.33 & $\mathrm{~T}$ \\
\hline Total number of esters (40) & 18 & & 18 & & 30 & \\
\hline \multicolumn{7}{|l|}{ Alkanes } \\
\hline$n$-pentane & & ND & 17.80 & $\mathrm{P}$ & & ND \\
\hline 2-methylpentane & & ND & 18.45 & $\mathrm{~T}$ & & ND \\
\hline 3-methylpentane & & ND & 18.75 & $\mathrm{~T}$ & & ND \\
\hline$n$-hexane & 22.76 & $\mathrm{P}$ & 19.00 & $\mathrm{P}$ & 21.43 & $\mathrm{~T}$ \\
\hline 2-methylhexane & & ND & 20.04 & $\mathrm{~T}$ & & ND \\
\hline 3-methylhexane & & ND & 20.85 & $\mathrm{~T}$ & & ND \\
\hline cyclohexane & & ND & 21.00 & $\mathrm{~T}$ & & ND \\
\hline$n$-heptane & 24.31 & $\mathrm{P}$ & 21.70 & $\mathrm{P}$ & 24.52 & $\mathrm{~T}$ \\
\hline 1,3-dimethylcyclopentane & & ND & 22.35 & $\mathrm{~T}$ & & ND \\
\hline 1,2-dimethylcyclopentane & & ND & 22.65 & $\mathrm{~T}$ & & ND \\
\hline methylcyclohexane & & ND & 25.60 & $\mathrm{~T}$ & & ND \\
\hline$n$-octane & 29.34 & $\mathrm{P}$ & 27.95 & $P$ & 32.57 & $\mathrm{~T}$ \\
\hline 2,2-dimethylbutane & 70.38 & $\mathrm{~T}$ & & ND & & ND \\
\hline 5-methylundecane & 112.14 & $\mathrm{~T}$ & & ND & & ND \\
\hline$n$-tetradecane & & ND & 116.70 & $\mathrm{~T}$ & & ND \\
\hline \multicolumn{7}{|l|}{ Aromatic hydrocarbons } \\
\hline benzene & 46.62 & $P$ & & ND & & ND \\
\hline toluene & 63.55 & $\mathrm{P}$ & & ND & 107.29 & $\mathrm{~T}$ \\
\hline styrene & & ND & 102.10 & $\mathrm{~T}$ & & ND \\
\hline ethyl benzene & & ND & & ND & 134.90 & $\mathrm{~T}$ \\
\hline$p$-xylene & & ND & & ND & 136.81 & $\mathrm{~T}$ \\
\hline$m$-xylene & 84.48 & $\mathrm{~T}$ & & ND & 138.62 & $\mathrm{~T}$ \\
\hline$o$-xylene & & ND & & ND & 150.43 & $\mathrm{~T}$ \\
\hline indene & 124.14 & $\mathrm{~T}$ & & ND & & ND \\
\hline naphthalene & 149.93 & $\mathrm{~T}$ & & ND & & ND \\
\hline \multicolumn{7}{|l|}{ Unsaturated hydrocarbons } \\
\hline 2-methyl-1-propene & 21.10 & $\mathrm{~T}$ & & ND & & ND \\
\hline
\end{tabular}


Table II. Continued.

\begin{tabular}{|c|c|c|c|c|c|c|}
\hline \multirow{2}{*}{ Compounds } & \multicolumn{2}{|c|}{ Method $A^{\text {a }}$} & \multicolumn{2}{|c|}{ Method $\boldsymbol{B}^{\mathrm{b}}$} & \multicolumn{2}{|c|}{ Method $C^{b}$} \\
\hline & $\mathrm{k}$ & I & $\mathrm{k}$ & I & $\mathrm{k}$ & $\mathrm{I}$ \\
\hline 1,3-pentadiene & & ND & 19.40 & $\mathrm{~T}$ & & ND \\
\hline 1,4-pentadiene & 23.24 & $\mathrm{~T}$ & & ND & & ND \\
\hline 1-heptene & 31.03 & $\mathrm{~T}$ & & ND & & ND \\
\hline 3-octene & 32.79 & $\mathrm{~T}$ & 29.65 & $\mathrm{~T}$ & 39.38 & $\mathrm{~T}$ \\
\hline 2-octene & 34.14 & $\mathrm{~T}$ & 32.95 & $\mathrm{~T}$ & & ND \\
\hline Total number of hydrocarbons (30) & 15 & & 17 & & 9 & \\
\hline \multicolumn{7}{|l|}{ Primary alcohols } \\
\hline ethanol & & ND & 44.50 & $\mathrm{P}$ & 64.71 & $\mathrm{P}$ \\
\hline 1-propanol & 64.24 & $\mathrm{~T}$ & 63.05 & $\mathrm{P}$ & 106.24 & $\mathrm{P}$ \\
\hline 2-propen-1-ol & & ND & 77.10 & $\mathrm{~T}$ & 130.29 & $\mathrm{~T}$ \\
\hline 1-butanol & 81.14 & $\mathrm{P}$ & 84.85 & $\mathrm{P}$ & 138.57 & $\mathrm{P}$ \\
\hline 1-pentanol & 95.45 & $\mathrm{~T}$ & & ND & 163.86 & $\mathrm{P}$ \\
\hline 1-hexanol & 108.28 & $\mathrm{P}$ & 112.50 & $\mathrm{~T}$ & 193.33 & $\mathrm{P}$ \\
\hline 1-heptanol & 125.86 & $\mathrm{~T}$ & & ND & & ND \\
\hline 1-octanol & & ND & 135.10 & $\mathrm{~T}$ & & ND \\
\hline \multicolumn{7}{|l|}{ Secondary alcohols } \\
\hline 2-propanol & 45.48 & $\mathrm{P}$ & 47.60 & $\mathrm{~T}$ & 61.05 & $\mathrm{P}$ \\
\hline 2-butanol & 61.72 & $\mathrm{P}$ & 60.95 & $\mathrm{P}$ & 102.33 & $\mathrm{P}$ \\
\hline 2-pentanol & 77.52 & $\mathrm{P}$ & 77.50 & $\mathrm{P}$ & 131.62 & $\mathrm{P}$ \\
\hline 2-hexanol & 98.34 & $\mathrm{~T}$ & 95.35 & $\mathrm{~T}$ & 156.81 & $\mathrm{P}$ \\
\hline 2-heptanol & 103.93 & $\mathrm{P}$ & 108.20 & $\mathrm{~T}$ & 181.67 & $\mathrm{P}$ \\
\hline \multicolumn{7}{|l|}{ Branched-chain alcohols } \\
\hline 2-methyl-1-propanol & & ND & 73.00 & $P$ & 124.00 & $\mathrm{P}$ \\
\hline 3-methyl-2-pentanol & & ND & 90.85 & $\mathrm{~T}$ & & ND \\
\hline 3-methyl-1-butanol & 90.24 & $\mathrm{P}$ & 93.40 & $\mathrm{P}$ & 150.05 & $\mathrm{P}$ \\
\hline 3-methyl-3-buten-1-ol & 95.69 & $\mathrm{~T}$ & 97.90 & $\mathrm{P}$ & 164.33 & $\mathrm{~T}$ \\
\hline 3-methyl-2-buten-1-ol & 104.52 & $\mathrm{~T}$ & 107.95 & $\mathrm{~T}$ & 183.43 & $\mathrm{~T}$ \\
\hline 2-ethyl-1-hexanol & 121.52 & $\mathrm{~T}$ & 128.00 & $\mathrm{~T}$ & & ND \\
\hline \multicolumn{7}{|l|}{ Oxy ether alcohols } \\
\hline 2-ethoxyethanol & 99.41 & $\mathrm{~T}$ & 95.75 & $\mathrm{~T}$ & & ND \\
\hline 2-butoxyethanol & 114.34 & $\mathrm{P}$ & 118.90 & $\mathrm{~T}$ & 222.00 & $\mathrm{~T}$ \\
\hline \multicolumn{7}{|l|}{ Aromatic alcohols } \\
\hline phenol & & ND & 178.90 & $\mathrm{~T}$ & 274.00 & $\mathrm{~T}$ \\
\hline 2-phenyl-1-ethanol & & ND & 170.85 & $\mathrm{~T}$ & & ND \\
\hline Total number of alcohols (23) & 16 & & 21 & & 17 & \\
\hline \multicolumn{7}{|l|}{$n$-Aldehydes } \\
\hline ethanal & 24.93 & $\mathrm{P}$ & 21.15 & $\mathrm{P}$ & 25.48 & $\mathrm{P}$ \\
\hline
\end{tabular}


Table II. Continued.

\begin{tabular}{|c|c|c|c|c|c|c|}
\hline \multirow{2}{*}{ Compounds } & \multicolumn{2}{|c|}{ Method $\boldsymbol{A}^{\mathrm{a}}$} & \multicolumn{2}{|c|}{ Method $\boldsymbol{B}^{\mathrm{b}}$} & \multicolumn{2}{|c|}{ Method $\boldsymbol{C}^{\mathrm{b}}$} \\
\hline & $\mathrm{k}$ & I & $\mathrm{k}$ & I & $\mathrm{k}$ & I \\
\hline propanal & & ND & 25.70 & $\mathrm{~T}$ & 33.14 & $\mathrm{~T}$ \\
\hline 2-propenal & & ND & 30.95 & $\mathrm{~T}$ & 41.67 & $\mathrm{~T}$ \\
\hline$n$-butanal & 37.41 & $\mathrm{~T}$ & 38.50 & $\mathrm{~T}$ & 47.43 & $\mathrm{P}$ \\
\hline$n$-pentanal & 62.28 & $\mathrm{~T}$ & & ND & & ND \\
\hline$n$-hexanal & 70.31 & $\mathrm{~T}$ & & ND & 121.43 & $\mathrm{P}$ \\
\hline$n$-heptanal & 86.45 & $\mathrm{~T}$ & & ND & & ND \\
\hline$n$-octanal & 108.76 & $\mathrm{~T}$ & & ND & & ND \\
\hline$n$-nonanal & 113.17 & $\mathrm{~T}$ & 116.75 & $\mathrm{P}$ & 210.00 & $\mathrm{P}$ \\
\hline$n$-decanal & 124.24 & $\mathrm{~T}$ & & ND & & ND \\
\hline \multicolumn{7}{|l|}{ Branched-chain aldehydes } \\
\hline 2-methylpropanal & & ND & 27.25 & $\mathrm{~T}$ & 35.81 & $\mathrm{~T}$ \\
\hline 2-methylbutanal & & ND & & ND & 56.90 & $\mathrm{~T}$ \\
\hline 3-methylbutanal & & ND & 40.30 & $\mathrm{P}$ & 58.24 & $\mathrm{P}$ \\
\hline \multicolumn{7}{|l|}{ Aromatic aldehydes } \\
\hline phenylethanal & ND & & ND & & 241.86 & $\mathrm{~T}$ \\
\hline Total number of aldehydes (14) & 8 & & 7 & & 10 & \\
\hline \multicolumn{7}{|l|}{ Methyl ketones } \\
\hline 2-propanone & 30.93 & $\mathrm{~T}$ & 27.7 & $\mathrm{P}$ & 36.38 & $\mathrm{P}$ \\
\hline 2-butanone & 41.17 & $\mathrm{P}$ & 39.10 & $\mathrm{P}$ & 53.23 & $\mathrm{P}$ \\
\hline 3-buten-2-one & 48.28 & $\mathrm{~T}$ & 51.35 & $\mathrm{~T}$ & & ND \\
\hline 2-pentanone & 53.31 & $\mathrm{P}$ & 51.55 & $P$ & 81.95 & $\mathrm{P}$ \\
\hline 3-methyl-2-pentanone & & ND & 62.45 & $\mathrm{~T}$ & & ND \\
\hline 4-methyl-2-pentanone & 59.28 & $\mathrm{~T}$ & & ND & & ND \\
\hline 2-hexanone & 70.10 & $\mathrm{P}$ & 73.90 & $\mathrm{P}$ & & ND \\
\hline 2-heptanone & 85.90 & $\mathrm{P}$ & 86.75 & $\mathrm{P}$ & 149.29 & $\mathrm{P}$ \\
\hline 2-octanone & 100.10 & $\mathrm{~T}$ & & ND & & ND \\
\hline 3-hydroxy-2-butanone & 101.24 & $\mathrm{~T}$ & 104.00 & $\mathrm{P}$ & 176.00 & $\mathrm{P}$ \\
\hline 2-nonanone & 112.31 & $\mathrm{P}$ & 117.40 & $\mathrm{P}$ & 208.52 & $\mathrm{P}$ \\
\hline \multicolumn{7}{|l|}{ Propyl ketones } \\
\hline 4-nonanone & 104.72 & $\mathrm{~T}$ & & ND & & ND \\
\hline \multicolumn{7}{|l|}{ Aromatic ketones } \\
\hline 1-phenylethanone & 140.79 & $\mathrm{~T}$ & & ND & 242.48 & $\mathrm{~T}$ \\
\hline \multicolumn{7}{|l|}{ Diketones } \\
\hline 2,3-butanedione & & ND & 52.45 & $\mathrm{P}$ & 83.27 & $\mathrm{P}$ \\
\hline Total number of ketones (14) & 12 & & 10 & & 8 & \\
\hline \multicolumn{7}{|l|}{$n$-Acids } \\
\hline ethanoic acid & 118.48 & $\mathrm{P}$ & 125.60 & $\mathrm{P}$ & 221.00 & $\mathrm{~T}$ \\
\hline
\end{tabular}


Table II. Continued.

\begin{tabular}{|c|c|c|c|c|c|c|}
\hline \multirow{2}{*}{ Compounds } & \multicolumn{2}{|c|}{ Method $\boldsymbol{A}^{\mathrm{a}}$} & \multicolumn{2}{|c|}{ Method $\boldsymbol{B}^{\mathrm{b}}$} & \multicolumn{2}{|c|}{ Method $C^{b}$} \\
\hline & $\mathrm{k}$ & I & $\mathrm{k}$ & I & $\mathrm{k}$ & I \\
\hline$n$-propanoic acid & & ND & 134.10 & $\mathrm{P}$ & & ND \\
\hline$n$-butanoic acid & 135.76 & $\mathrm{P}$ & 144.15 & $\mathrm{P}$ & 238.95 & $\mathrm{~T}$ \\
\hline$n$-pentanoic acid & 145.68 & $\mathrm{P}$ & & ND & & ND \\
\hline$n$-hexanoic acid & 155.41 & $\mathrm{P}$ & 164.65 & $\mathrm{~T}$ & 251.48 & $\mathrm{~T}$ \\
\hline$n$-octanoic acid & & ND & 180.95 & $\mathrm{~T}$ & & ND \\
\hline \multicolumn{7}{|l|}{ Branched-chain acids } \\
\hline 2-methylpropanoic acid & 129.97 & $\mathrm{P}$ & 137.95 & $\mathrm{P}$ & 233.81 & $\mathrm{~T}$ \\
\hline 3-methylbutanoic acid & 139.66 & $\mathrm{P}$ & 148.20 & $\mathrm{P}$ & 241.62 & $\mathrm{~T}$ \\
\hline 2-methylbutanoic acid & 146.24 & $\mathrm{~T}$ & & ND & & ND \\
\hline 2-ethylhexanoic acid & & ND & 173.55 & $\mathrm{~T}$ & & ND \\
\hline Total number of acids (10) & 7 & & 8 & & 5 & \\
\hline \multicolumn{7}{|l|}{ Sulphur compounds } \\
\hline carbon disulphide & & ND & 21.90 & $\mathrm{P}$ & 27.52 & $\mathrm{~T}$ \\
\hline dimethyl sulphide & & ND & & ND & 28.91 & $\mathrm{~T}$ \\
\hline dimethyl disulphide & & ND & 66.90 & $\mathrm{~T}$ & 119.14 & $\mathrm{~T}$ \\
\hline $\begin{array}{l}\text { Total number of sulphur } \\
\text { compounds (3) }\end{array}$ & $\mathbf{0}$ & & 2 & & 3 & \\
\hline \multicolumn{7}{|l|}{ Terpenes } \\
\hline$\alpha$-pinene & & ND & 63.00 & $\mathrm{~T}$ & 98.29 & $\mathrm{P}$ \\
\hline$D$-limonene & 86.93 & $\mathrm{~T}$ & 91.50 & $\mathrm{P}$ & 153.10 & $\mathrm{P}$ \\
\hline$p$-cymene & 106.79 & $\mathrm{~T}$ & & ND & 171.71 & $\mathrm{~T}$ \\
\hline Total number of terpenes (3) & 2 & & 2 & & 3 & \\
\hline $\begin{array}{l}\text { Total number of volatile } \\
\text { compounds (137) }\end{array}$ & 78 & & 85 & & 84 & \\
\hline
\end{tabular}

a Method using P\&T-MWH.

b Methods using P\&T-RH; k: retention factor; I: identification; P: positively identified by comparison with $\mathrm{k}$ and mass spectra of authentic standards; $\mathrm{T}$ : tentatively identified on the basis of the mass spectra libraries; ND: not detected.

according to the DHS method used. Only 8 of the 40 esters, and 4 of the 30 hydrocarbons were detected by the three methods, whereas 22 esters and 23 hydrocarbons were detected by only one of the methods (not always the same one). A larger number of individual esters was detected with method $\boldsymbol{C}$ compared with methods $\boldsymbol{A}$ and $\boldsymbol{B}$, probably due to the higher purge temperature used in method $C$ (at $50^{\circ} \mathrm{C}$ ) compared with methods $\boldsymbol{A}$ and $\boldsymbol{B}$ (both at $40^{\circ} \mathrm{C}$ ). The largest numbers of individual hydrocarbons were detected by methods $\boldsymbol{A}$ and $\boldsymbol{B}$ (15 and 17 hydrocarbons, respectively) (Tab. II).

Alcohols and acids also showed variations according to the DHS method used. Only 12 of the 23 alcohols, and 5 of the 10 acids were detected by each of the three methods, whereas 4 alcohols and 5 acids were detected by only one of the methods (not always the same one). The largest number of individual alcohols was detected by method $\boldsymbol{B}$. It was remarkable that all secondary alcohols were detected by the three 
methods, and that no aromatic alcohol was detected by method $\boldsymbol{A}$. The total number of acids detected by each of the three methods was between 5 and 8 (Tab. II).

Only 9 of the 28 carbonyl compounds were detected by the three methods, whereas 6 aldehydes and 4 ketones were detected by only one of the methods (not always the same one). Most individual carbonyl compounds were methyl ketones and $n$-aldehydes. The total number of aldehydes detected by each of the three methods was between 7 and 10, and that for ketones was between 8 and 12 (Tab. II). No sulphur compounds were detected by method $\boldsymbol{A}$, whereas all terpenes were always detected at least by two of the three methods (Tab. II).

Tables III-VII list the mean percentages of the volatile compounds, grouped by chemical families, which were found in the headspace of the five PDO cheeses analysed by each of the three methods. Ethanoic acid, $n$-butanoic acid and 2-butanol were quantified individually because of their large percentage found in some of the cheese varieties. Large differences in the percentages of the volatile compounds of each type of cheese were recorded between the method $\boldsymbol{A}$ which used P\&T-MHW and the other two methods $\boldsymbol{B}$ and $\boldsymbol{C}$ which used P\&T-RH. Large percentages of acids in Idiazabal (90.3\%), Roncal (80.6\%), and Zamorano $(77.9 \%)$ cheeses were found with method $\boldsymbol{A}$, whereas large percentages of alcohols (72-92\%) were found with methods $\boldsymbol{B}$ and $\boldsymbol{C}$ in the same cheeses (Tabs. III-V). As has been previously published, considerable amounts of short-chain free fatty acids have been reported in ripened Idiazabal and Roncal cheeses. Several authors have reported that the content of short-chain fatty acids in Idiazabal cheese after six months of ripening was around 20 $30 \%$ of the total concentration of free fatty acids $[9,10]$, and that short-chain fatty acids were the main volatile compounds analysed by simultaneous distillation extraction (SDE) in Roncal cheese after five months of ripening [27]. The percentage of acids was also higher when the volatiles of Manchego and La Serena cheeses were analysed by P\&T-MWH (method $\boldsymbol{A})$ compared with P\&T-RH (methods $\boldsymbol{B}$ and $\boldsymbol{C}$ ) (Tabs. VI and VII). It has been reported that short-chain free fatty acids were present in low concentrations in ripened Manchego cheese [44], and this is in agreement with the lower percentage of acids found in this PDO cheese (9.4\%) by method $\boldsymbol{A}$. Although no data on free fatty acid composition of Zamorano and La Serena cheeses has been published, it could be indicated that the extraction of volatile acids by semi-automatic P\&TMWH (used in method $\boldsymbol{A}$ ) was larger than that of automatic P\&T-RH (used in methods $\boldsymbol{B}$ and $\boldsymbol{C}$ ). These results could be explained by the different nature of the trap absorbent. The graphitised carbon used by method $\boldsymbol{A}$ shows high specific surface area with a large number of active sites for polar compounds. Thus, polar volatile compounds such as short-chain fatty acids are strongly retained in its surface. However, tenax which was used by methods $\boldsymbol{B}$ anc $\boldsymbol{C}$ is a hydrophobic porous polymer with low specific surface area [40]. On the other hand, it must be indicated that tenax is not suitable for desorption by MWH because of its poor heating capability by microwaves [43].

The three methods found large percentages of alcohols in Manchego cheese, although significant differences $(P \leq 0.05)$ were recorded among them $(61.7,81.8$, and $94.3 \%$ of total alcohols, respectively, for methods $\boldsymbol{A}, \boldsymbol{B}$ and $\boldsymbol{C}$ ).

A large percentage of aldehydes $(58.4 \%)$ was found by method $\boldsymbol{A}$ in La Serena cheese, whereas large percentages of alcohols (around 85\%) were found in this type of cheese with methods $\boldsymbol{B}$ and $\boldsymbol{C}$. It was remarkable that aldehydes constituted the main volatile compounds of the aroma profile of La Serena cheese when volatiles were analysed by P\&T-MWH (Tab. VII). The percentages of aldehydes found in the other PDO cheeses were also higher when volatile compounds were analysed with method $\boldsymbol{A}$ compared with methods $\boldsymbol{B}$ and $\boldsymbol{C}$ (Tabs. III-VI) because the aldehydes were strongly retained by graphitised carbon.

In general, larger percentages of ketones were detected by methods $\boldsymbol{B}$ and $\boldsymbol{C}$ than by method $\boldsymbol{A}$, whereas larger percentages of esters were found in the PDO cheese varieties with method A, compared with methods $\boldsymbol{B}$ and $\boldsymbol{C}$. Except for Manchego cheese, the percentages of hydrocarbons in the PDO cheese varieties found by each of the 
Table III. Percentage (means $\pm \mathrm{SD} ; n=4$ ) of the volatile compounds found in Idiazabal cheese using different DHS methods.

\begin{tabular}{|c|c|c|c|}
\hline & Method $A^{\mathrm{a}}$ & Method $\boldsymbol{B}^{\mathrm{b}}$ & Method $C^{b}$ \\
\hline ethanoic acid & $1.48 \pm 0.39^{\mathrm{a}}$ & $1.78 \pm 0.90^{\mathrm{a}}$ & $0.01 \pm 0.00^{b}$ \\
\hline$n$-butanoic acid & $65.55 \pm 5.78^{\mathrm{a}}$ & $1.62 \pm 0.66^{\mathrm{b}}$ & $0.03 \pm 0.01^{b}$ \\
\hline$n$-acids & $6.17 \pm 0.91^{\mathrm{a}}$ & $0.20 \pm 0.12^{b}$ & $0.01 \pm 0.00^{\mathrm{b}}$ \\
\hline branched-chain acids & $17.06 \pm 5.34^{\mathrm{a}}$ & $2.03 \pm 0.71^{b}$ & $0.05 \pm 0.02^{b}$ \\
\hline Total acids & $90.27 \pm 2.47^{a}$ & $5.62 \pm 2.39 \mathrm{~b}$ & $0.10 \pm 0.03 c$ \\
\hline ethanol & $\mathrm{ND}^{\mathrm{a}}$ & $8.19 \pm 0.82^{b}$ & $2.23 \pm 0.46^{\mathrm{c}}$ \\
\hline 2-butanol & $1.88 \pm 1.04^{\mathrm{a}}$ & $66.81 \pm 2.91^{b}$ & $81.52 \pm 1.15^{\mathrm{c}}$ \\
\hline primary alcohols & $2.81 \pm 0.63^{\mathrm{a}}$ & $7.21 \pm 0.91^{b}$ & $5.93 \pm 0.43^{b}$ \\
\hline secondary alcohols & $0.18 \pm 0.07^{\mathrm{a}}$ & $1.55 \pm 0.31^{\mathrm{b}}$ & $1.80 \pm 0.16^{b}$ \\
\hline branched-chain alcohols & $0.10 \pm 0.05^{\mathrm{a}}$ & $0.18 \pm 0.02^{\mathrm{a}}$ & $0.36 \pm 0.05^{b}$ \\
\hline oxy ether alcohols & $0.72 \pm 0.27 \mathrm{a}$ & $0.05 \pm 0.00^{\mathrm{b}}$ & $0.07 \pm 0.01^{b}$ \\
\hline aromatic alcohols & $\mathrm{ND}^{\mathrm{a}}$ & $\mathrm{ND}^{\mathrm{a}}$ & $\mathrm{ND}^{\mathrm{a}}$ \\
\hline Total alcohols & $5.70 \pm 1.20^{\mathrm{a}}$ & $83.99 \pm 3.31^{b}$ & $91.90 \pm 1.27^{c}$ \\
\hline$n$-aldehydes & $0.76 \pm 0.25^{\mathrm{a}}$ & $0.11 \pm 0.01^{b}$ & $0.08 \pm 0.01^{b}$ \\
\hline branched-chain aldehydes & $\mathrm{ND}^{\mathrm{a}}$ & $0.06 \pm 0.02^{b}$ & $0.11 \pm 0.03^{\mathrm{c}}$ \\
\hline aromatic aldehydes & $\mathrm{ND}^{\mathrm{a}}$ & $\mathrm{ND}^{\mathrm{a}}$ & $\mathrm{ND}^{\mathrm{a}}$ \\
\hline Total aldehydes & $0.76 \pm 0.25^{a}$ & $0.16 \pm 0.02^{b}$ & $0.19 \pm 0.02^{b}$ \\
\hline methyl ketones & $0.08 \pm 0.04^{\mathrm{a}}$ & $8.71 \pm 1.31^{b}$ & $4.73 \pm 1.38^{\mathrm{c}}$ \\
\hline propyl ketones & $\mathrm{ND}^{\mathrm{a}}$ & $\mathrm{ND}^{\mathrm{a}}$ & $\mathrm{ND}^{\mathrm{a}}$ \\
\hline aromatic ketones & $\mathrm{ND}^{\mathrm{a}}$ & $\mathrm{ND}^{\mathrm{a}}$ & $<0.01^{\mathrm{b}}$ \\
\hline diketones & $\mathrm{ND}^{\mathrm{a}}$ & $0.05 \pm 0.10^{\mathrm{b}}$ & $0.08 \pm 0.01^{b}$ \\
\hline Total ketones & $0.08 \pm 0.04 a^{a}$ & $8.77 \pm 1.31^{b}$ & $4.82 \pm 1.40^{c}$ \\
\hline methyl esters & $\mathrm{ND}^{\mathrm{a}}$ & $\mathrm{ND}^{\mathrm{a}}$ & $0.01 \pm 0.00^{b}$ \\
\hline ethyl esters & $0.44 \pm 0.17^{\mathrm{a}}$ & $0.17 \pm 0.02^{\mathrm{a}}$ & $0.95 \pm 0.02^{b}$ \\
\hline propyl esters & $0.10 \pm 0.10^{\mathrm{a}}$ & $0.02 \pm 0.00^{\mathrm{a}}$ & $0.58 \pm 0.04^{\mathrm{b}}$ \\
\hline butyl esters & $0.50 \pm 0.07^{\mathrm{a}}$ & $0.04 \pm 0.01^{b}$ & $0.13 \pm 0.03^{c}$ \\
\hline higher alkyl esters & $0.37 \pm 0.50^{\mathrm{a}}$ & $\mathrm{ND}^{\mathrm{b}}$ & $\mathrm{ND}^{\mathrm{b}}$ \\
\hline branched alkyl esters & $1.10 \pm 0.63^{\mathrm{a}}$ & $0.29 \pm 0.02^{b}$ & $0.90 \pm 0.08^{\mathrm{ab}}$ \\
\hline Total esters & $2.50 \pm 1.20^{\mathrm{a}}$ & $0.52 \pm 0.04^{b}$ & $2.57 \pm 0.16^{\mathrm{a}}$ \\
\hline alkanes & $0.67 \pm 0.57 \mathrm{ab}$ & $0.84 \pm 0.11^{\mathrm{a}}$ & $0.06 \pm 0.00^{b}$ \\
\hline aromatic hydrocarbons & $0.04 \pm 0.01^{\mathrm{a}}$ & $0.01 \pm 0.01^{\mathrm{a}}$ & $0.23 \pm 0.13^{b}$ \\
\hline unsaturated hydrocarbons & $\mathrm{ND}^{\mathrm{a}}$ & $0.06 \pm 0.01^{b}$ & $0.09 \pm 0.00^{c}$ \\
\hline Total hydrocarbons & $0.70 \pm 0.57^{a}$ & $0.91 \pm 0.11^{\mathrm{a}}$ & $0.38 \pm 0.13^{a}$ \\
\hline sulphur compounds & $\mathrm{ND}^{\mathrm{a}}$ & $0.03 \pm 0.01^{b}$ & $0.03 \pm 0.10^{\mathrm{b}}$ \\
\hline terpenes & $\mathrm{ND}^{\mathrm{a}}$ & $\mathrm{ND}^{\mathrm{a}}$ & $0.01 \pm 0.01^{b}$ \\
\hline
\end{tabular}

a Method using P\&T-MWH. ${ }^{\mathrm{b}}$ Methods using P\&T-RH. ND: not detected.

${ }^{\mathrm{a}-\mathrm{c}}$ Means within rows without a common superscript are significantly different $(P \leq 0.05)$. 
Table IV. Percentage (means $\pm \mathrm{SD} ; n=4$ ) of the volatile compounds found in Roncal cheese using different DHS methods.

\begin{tabular}{|c|c|c|c|}
\hline & Method $A^{\text {a }}$ & Method $\boldsymbol{B}^{\mathrm{b}}$ & Method $C^{b}$ \\
\hline ethanoic acid & $3.17 \pm 0.64^{\mathrm{a}}$ & $0.43 \pm 0.13^{b}$ & $<0.01^{b}$ \\
\hline$n$-butanoic acid & $37.35 \pm 2.47^{a}$ & $0.67 \pm 0.05^{b}$ & $0.01 \pm 0.00^{\mathrm{b}}$ \\
\hline$n$-acids & $32.27 \pm 1.07^{\mathrm{a}}$ & $0.06 \pm 0.02^{b}$ & $\mathrm{ND}^{\mathrm{b}}$ \\
\hline branched-chain acids & $7.82 \pm 3.51^{\mathrm{a}}$ & $1.78 \pm 0.83^{b}$ & $<0.01^{\mathrm{b}}$ \\
\hline Total acids & $80.62 \pm 0.96^{a}$ & $2.93 \pm 0.76^{b}$ & $0.01 \pm 0.00^{c}$ \\
\hline ethanol & $\mathrm{ND}^{\mathrm{a}}$ & $11.01 \pm 1.66^{\mathrm{b}}$ & $3.33 \pm 0.55^{\mathrm{c}}$ \\
\hline 2-butanol & $9.06 \pm 1.18^{\mathrm{a}}$ & $53.28 \pm 2.66^{\mathrm{b}}$ & $73.97 \pm 1.81^{\mathrm{c}}$ \\
\hline primary alcohols & $1.52 \pm 0.39^{\mathrm{a}}$ & $5.60 \pm 0.61^{b}$ & $4.47 \pm 0.55^{\mathrm{c}}$ \\
\hline secondary alcohols & $\mathrm{ND}^{\mathrm{a}}$ & $1.26 \pm 0.27^{b}$ & $1.34 \pm 0.14^{b}$ \\
\hline branched-chain alcohols & $0.71 \pm 0.45^{\mathrm{a}}$ & $0.170 \pm 0.16^{\mathrm{a}}$ & $0.18 \pm 0.02^{\mathrm{a}}$ \\
\hline oxy ether alcohols & $0.52 \pm 0.24^{\mathrm{a}}$ & $0.06 \pm 0.06^{b}$ & $0.02 \pm 0.00^{\mathrm{b}}$ \\
\hline aromatic alcohols & $\mathrm{ND}^{\mathrm{a}}$ & $0.14 \pm 0.26^{\mathrm{b}}$ & $\mathrm{ND}^{\mathrm{a}}$ \\
\hline Total alcohols & $11.80 \pm 1.62^{\mathrm{a}}$ & $71.52 \pm 2.77^{b}$ & $83.30 \pm 2.79^{c}$ \\
\hline$n$-aldehydes & $1.26 \pm 0.50^{\mathrm{a}}$ & $0.10 \pm 0.02^{b}$ & $0.19 \pm 0.02^{b}$ \\
\hline branched-chain aldehydes & $\mathrm{ND}^{\mathrm{a}}$ & $0.08 \pm 0.06^{\mathrm{b}}$ & $0.15 \pm 0.02^{b}$ \\
\hline aromatic aldehydes & $\mathrm{ND}^{\mathrm{a}}$ & $\mathrm{ND}^{\mathrm{a}}$ & $0.01 \pm 0.00^{\mathrm{b}}$ \\
\hline Total aldehydes & $1.26 \pm 0.50^{\mathrm{a}}$ & $0.18 \pm 0.08^{b}$ & $0.35 \pm 0.05^{b}$ \\
\hline methyl ketones & $1.28 \pm 0.56^{\mathrm{a}}$ & $23.68 \pm 3.93^{b}$ & $15.21 \pm 2.71^{\mathrm{c}}$ \\
\hline propyl ketones & $\mathrm{ND}^{\mathrm{a}}$ & $\mathrm{ND}^{\mathrm{a}}$ & $\mathrm{ND}^{\mathrm{a}}$ \\
\hline aromatic ketones & $\mathrm{ND}^{\mathrm{a}}$ & $\mathrm{ND}^{\mathrm{a}}$ & $<0.01^{b}$ \\
\hline diketones & $\mathrm{ND}^{\mathrm{a}}$ & $0.32 \pm 0.23^{b}$ & $0.17 \pm 0.05^{b}$ \\
\hline Total ketones & $1.28 \pm 0.56^{a}$ & $24.00 \pm 3.86^{b}$ & $15.38 \pm 2.76^{c}$ \\
\hline methyl esters & $\mathrm{ND}^{\mathrm{a}}$ & $0.02 \pm 0.01 \mathrm{~b}$ & $0.01 \pm 0.01^{b}$ \\
\hline ethyl esters & $0.47 \pm 0.30^{\mathrm{a}}$ & $0.11 \pm 0.03^{b}$ & $0.26 \pm 0.03^{\mathrm{ab}}$ \\
\hline propyl esters & $\mathrm{ND}^{\mathrm{a}}$ & $\mathrm{ND}^{\mathrm{a}}$ & $0.14 \pm 0.03^{b}$ \\
\hline butyl esters & $0.45 \pm 0.16^{\mathrm{a}}$ & $\mathrm{ND}^{\mathrm{b}}$ & $0.01 \pm 0.00^{c}$ \\
\hline higher alkyl esters & $\mathrm{ND}^{\mathrm{a}}$ & $\mathrm{ND}^{\mathrm{a}}$ & $\mathrm{ND}^{\mathrm{a}}$ \\
\hline branched alkyl esters & $0.37 \pm 0.10^{\mathrm{a}}$ & $0.06 \pm 0.02^{b}$ & $0.11 \pm 0.03^{b}$ \\
\hline Total esters & $1.29 \pm 0.26^{a}$ & $0.19 \pm 0.02^{b}$ & $0.52 \pm 0.10^{c}$ \\
\hline alkanes & $3.42 \pm 1.05^{\mathrm{a}}$ & $1.09 \pm 0.71^{b}$ & $0.12 \pm 0.06^{\mathrm{b}}$ \\
\hline aromatic hydrocarbons & $0.34 \pm 0.11^{\mathrm{a}}$ & $\mathrm{ND}^{\mathrm{b}}$ & $0.21 \pm 0.05^{\mathrm{a}}$ \\
\hline unsaturated hydrocarbons & $\mathrm{ND}^{\mathrm{a}}$ & $0.02 \pm 0.01^{b}$ & $0.04 \pm 0.01^{\mathrm{c}}$ \\
\hline Total hydrocarbons & $3.75 \pm 0.96 \mathrm{a}$ & $1.11 \pm 0.71^{b}$ & $0.37 \pm \mathbf{0 . 0 8}^{b}$ \\
\hline sulphur compounds & $\mathrm{ND}^{\mathrm{a}}$ & $0.03 \pm 0.02^{b}$ & $0.02 \pm 0.00^{\mathrm{b}}$ \\
\hline terpenes & $\mathrm{ND}^{\mathrm{a}}$ & $0.03 \pm 0.01^{b}$ & $0.05 \pm 0.01^{\mathrm{c}}$ \\
\hline
\end{tabular}

a Method using P\&T-MWH. ${ }^{\mathrm{b}}$ Methods using P\&T-RH. ND: not detected.

a-c Means within rows without a common superscript are significantly different $(P \leq 0.05)$. 
Table V. Percentage (means $\pm \mathrm{SD} ; n=4$ ) of the volatile compounds found in Zamorano cheese using different DHS methods.

\begin{tabular}{|c|c|c|c|}
\hline & Method $A^{\text {a }}$ & Method $\boldsymbol{B}^{\mathrm{b}}$ & Method $C^{b}$ \\
\hline ethanoic acid & $33.75 \pm 8.25^{\mathrm{a}}$ & $3.08 \pm 0.74 b$ & $0.02 \pm 0.00^{\mathrm{b}}$ \\
\hline$n$-butanoic acid & $31.74 \pm 7.61^{\mathrm{a}}$ & $2.23 \pm 0.39 \mathrm{~b}$ & $0.02 \pm 0.00^{\mathrm{b}}$ \\
\hline$n$-acids & $8.03 \pm 2.07^{\mathrm{a}}$ & $0.27 \pm 0.05^{\mathrm{b}}$ & $\mathrm{ND}^{\mathrm{c}}$ \\
\hline branched-chain acids & $4.36 \pm 0.88^{\mathrm{a}}$ & $2.17 \pm 0.35^{b}$ & $\mathrm{ND}^{\mathrm{c}}$ \\
\hline Total acids & $77.88 \pm 6.62^{\mathrm{a}}$ & $7.74 \pm 1.49 \mathrm{~b}$ & $0.04 \pm 0.01^{c}$ \\
\hline ethanol & $\mathrm{ND}^{\mathrm{a}}$ & $28.25 \pm 2.08^{b}$ & $12.36 \pm 0.60^{\mathrm{c}}$ \\
\hline 2-butanol & $8.15 \pm 3.26^{\mathrm{a}}$ & $35.74 \pm 3.04^{b}$ & $56.36 \pm 6.67 \mathrm{c}$ \\
\hline primary alcohols & $0.80 \pm 0.06^{\mathrm{a}}$ & $2.80 \pm 0.36^{\mathrm{b}}$ & $1.69 \pm 0.54^{\mathrm{c}}$ \\
\hline secondary alcohols & $2.99 \pm 1.70^{\mathrm{a}}$ & $4.36 \pm 0.51^{\mathrm{ab}}$ & $5.62 \pm 0.50^{\mathrm{b}}$ \\
\hline branched-chain alcohols & $2.07 \pm 1.63^{\mathrm{a}}$ & $0.28 \pm 0.03^{b}$ & $0.62 \pm 0.07 \mathrm{ab}$ \\
\hline oxy ether alcohols & $0.49 \pm 0.19^{\mathrm{a}}$ & $0.05 \pm 0.00^{\mathrm{b}}$ & $\mathrm{ND}^{\mathrm{b}}$ \\
\hline aromatic alcohols & $\mathrm{ND}^{\mathrm{a}}$ & $\mathrm{ND}^{\mathrm{a}}$ & $\mathrm{ND}^{\mathrm{a}}$ \\
\hline Total alcohols & $14.49 \pm 5.56^{a}$ & $71.48 \pm 3.18^{b}$ & $76.65 \pm 6.44^{b}$ \\
\hline$n$-aldehydes & $2.53 \pm 0.46^{\mathrm{a}}$ & $0.27 \pm 0.02^{\mathrm{b}}$ & $0.32 \pm 0.10^{\mathrm{b}}$ \\
\hline branched-chain aldehydes & $\mathrm{ND}^{\mathrm{a}}$ & $0.19 \pm 0.07^{b}$ & $0.37 \pm 0.4^{\mathrm{c}}$ \\
\hline aromatic aldehydes & $\mathrm{ND}^{\mathrm{a}}$ & $\mathrm{ND}^{\mathrm{a}}$ & $0.20 \pm 0.01^{b}$ \\
\hline Total aldehydes & $2.53 \pm 0.46^{a}$ & $0.46 \pm 0.09^{b}$ & $0.71 \pm 0.14^{b}$ \\
\hline methyl ketones & $2.54 \pm 0.59^{\mathrm{a}}$ & $16.02 \pm 2.44^{b}$ & $12.57 \pm 1.96^{\mathrm{b}}$ \\
\hline propyl ketones & $\mathrm{ND}^{\mathrm{a}}$ & $\mathrm{ND}^{\mathrm{a}}$ & $\mathrm{ND}^{\mathrm{a}}$ \\
\hline aromatic ketones & $\mathrm{ND}^{\mathrm{a}}$ & $\mathrm{ND}^{\mathrm{a}}$ & $0.01 \pm 0.00^{\mathrm{b}}$ \\
\hline diketones & $\mathrm{ND}^{\mathrm{a}}$ & $0.43 \pm 0.15^{b}$ & $0.89 \pm 0.06^{\mathrm{c}}$ \\
\hline Total ketones & $2.54 \pm 0.59^{\mathrm{a}}$ & $16.45 \pm 2.55^{b}$ & $13.46 \pm 2.01^{b}$ \\
\hline methyl esters & $\mathrm{ND}^{\mathrm{a}}$ & $0.07 \pm 0.01^{\mathrm{b}}$ & $0.08 \pm 0.04^{b}$ \\
\hline ethyl esters & $1.76 \pm 0.75^{\mathrm{a}}$ & $0.39 \pm 0.05^{b}$ & $0.73 \pm 0.12^{b}$ \\
\hline propyl esters & $\mathrm{ND}^{\mathrm{a}}$ & $\mathrm{ND}^{\mathrm{a}}$ & $0.03 \pm 0.00^{b}$ \\
\hline butyl esters & $\mathrm{ND}^{\mathrm{a}}$ & $\mathrm{ND}^{\mathrm{a}}$ & $0.02 \pm 0.01^{b}$ \\
\hline higher alkyl esters & $\mathrm{ND}^{\mathrm{a}}$ & $\mathrm{ND}^{\mathrm{a}}$ & $\mathrm{ND}^{\mathrm{a}}$ \\
\hline branched alkyl esters & $0.21 \pm 0.08^{\mathrm{a}}$ & $0.08 \pm 0.01^{b}$ & $0.09 \pm 0.03^{b}$ \\
\hline Total esters & $0.96 \pm 0.81^{\mathrm{a}}$ & $0.54 \pm \mathbf{0 . 0 6}^{b}$ & $0.96 \pm 0.08^{b}$ \\
\hline alkanes & $0.60 \pm 0.18^{\mathrm{a}}$ & $2.42 \pm 0.76^{\mathrm{a}}$ & $7.37 \pm 8.43^{\mathrm{a}}$ \\
\hline aromatic hydrocarbons & $\mathrm{ND}^{\mathrm{a}}$ & $0.01 \pm 0.00^{\mathrm{b}}$ & $0.58 \pm 0.13^{c}$ \\
\hline unsaturated hydrocarbons & $\mathrm{ND}^{\mathrm{a}}$ & $0.08 \pm 0.02^{b}$ & $0.10 \pm 0.01^{b}$ \\
\hline Total hydrocarbons & $0.60 \pm 0.18^{a}$ & $2.52 \pm 0.75^{a}$ & $8.05 \pm 8.30^{\mathrm{a}}$ \\
\hline sulphur compounds & $\mathrm{ND}^{\mathrm{a}}$ & $0.82 \pm 0.25^{\mathrm{b}}$ & $0.09 \pm 0.01^{\mathrm{c}}$ \\
\hline terpenes & $\mathrm{ND}^{\mathrm{a}}$ & $\mathrm{ND}^{\mathrm{a}}$ & $0.03 \pm 0.01^{b}$ \\
\hline
\end{tabular}

a Method using P\&T-MWH. ${ }^{\mathrm{b}}$ Methods using P\&T-RH. ND: not detected.

a-c Means within rows without a common superscript are significantly different $(P \leq 0.05)$. 
Table VI. Percentage (means $\pm \mathrm{SD} ; n=4$ ) of the volatile compounds found in Manchego using different DHS methods.

\begin{tabular}{|c|c|c|c|}
\hline & Method $A^{\text {a }}$ & Method $\boldsymbol{B}^{\mathrm{b}}$ & Method $C^{b}$ \\
\hline ethanoic acid & $1.46 \pm 0.94^{\mathrm{a}}$ & $1.01 \pm 0.59^{\mathrm{a}}$ & $<0.01^{\mathrm{b}}$ \\
\hline$n$-butanoic acid & $5.50 \pm 4.50^{\mathrm{a}}$ & $0.84 \pm 0.43^{\mathrm{ab}}$ & $0.01 \pm 0.00^{\mathrm{b}}$ \\
\hline$n$-acids & $\mathrm{ND}^{\mathrm{a}}$ & $0.11 \pm 0.04^{b}$ & $\mathrm{ND}^{\mathrm{a}}$ \\
\hline branched-chain acids & $2.4 \pm 2.16^{\mathrm{a}}$ & $0.93 \pm 0.49^{\mathrm{a}}$ & $<0.01^{\mathrm{a}}$ \\
\hline Total acids & $9.37 \pm 5.70^{\mathrm{a}}$ & $2.88 \pm 1.55^{\mathrm{ab}}$ & $0.01 \pm 0.00^{b}$ \\
\hline ethanol & $\mathrm{ND}^{\mathrm{a}}$ & $12.07 \pm 0.80^{\mathrm{b}}$ & $3.57 \pm 0.42^{\mathrm{c}}$ \\
\hline 2-butanol & $48.90 \pm 22.72^{\mathrm{a}}$ & $59.61 \pm 3.40^{\mathrm{ab}}$ & $80.36 \pm 0.65^{b}$ \\
\hline primary alcohols & $7.98 \pm 3.94^{\mathrm{a}}$ & $5.22 \pm 0.80^{\mathrm{a}}$ & $4.35 \pm 0.29^{\mathrm{a}}$ \\
\hline secondary alcohols & $3.14 \pm 1.72^{\mathrm{a}}$ & $4.84 \pm 0.50^{\mathrm{ab}}$ & $5.61 \pm 0.42^{b}$ \\
\hline branched-chain alcohols & $0.77 \pm 0.41^{\mathrm{a}}$ & $0.13 \pm 0.02^{b}$ & $0.30 \pm 0.01^{\mathrm{ab}}$ \\
\hline oxy ether alcohols & $0.89 \pm 0.82^{\mathrm{a}}$ & $0.02 \pm 0.01^{\mathrm{a}}$ & $0.05 \pm 0.01^{\mathrm{a}}$ \\
\hline aromatic alcohols & $\mathrm{ND}^{\mathrm{a}}$ & $\mathrm{ND}^{\mathrm{a}}$ & $\mathrm{ND}^{\mathrm{a}}$ \\
\hline Total alcohols & $61.68 \pm 19.25^{a}$ & $81.88 \pm 2.40^{\mathrm{ab}}$ & $94.25 \pm 0.45^{b}$ \\
\hline$n$-aldehydes & $3.00 \pm 1.97^{\mathrm{a}}$ & $0.12 \pm 0.02^{\mathrm{b}}$ & $0.08 \pm 0.02^{b}$ \\
\hline branched-chain aldehydes & $\mathrm{ND}^{\mathrm{a}}$ & $0.08 \pm 0.02^{b}$ & $0.07 \pm 0.00^{b}$ \\
\hline aromatic aldehydes & $\mathrm{ND}^{\mathrm{a}}$ & $\mathrm{ND}^{\mathrm{a}}$ & $0.01 \pm 0.00^{\mathrm{b}}$ \\
\hline Total aldehydes & $3.00 \pm 1.97^{a}$ & $0.19 \pm 0.01^{b}$ & $0.16 \pm 0.01^{b}$ \\
\hline methyl ketones & $1.84 \pm 1.65^{\mathrm{a}}$ & $14.11 \pm 3.52^{b}$ & $4.19 \pm 0.58^{\mathrm{a}}$ \\
\hline propyl ketones & $0.12 \pm 0.08^{a}$ & $\mathrm{ND}^{\mathrm{b}}$ & $\mathrm{ND}^{\mathrm{b}}$ \\
\hline aromatic ketones & $\mathrm{ND}^{\mathrm{a}}$ & $\mathrm{ND}^{\mathrm{a}}$ & $<0.01^{b}$ \\
\hline diketones & $\mathrm{ND}^{\mathrm{a}}$ & $0.10 \pm 0.04^{b}$ & $0.05 \pm 0.02^{b}$ \\
\hline Total ketones & $1.96 \pm 1.60^{\mathrm{a}}$ & $14.21 \pm 3.56^{b}$ & $4.25 \pm 0.60^{\mathrm{a}}$ \\
\hline methyl esters & $\mathrm{ND}^{\mathrm{a}}$ & $0.02 \pm 0.01^{b}$ & $0.02 \pm 0.01^{b}$ \\
\hline ethyl esters & $2.43 \pm 1.68^{\mathrm{a}}$ & $0.08 \pm 0.01^{b}$ & $0.22 \pm 0.02^{b}$ \\
\hline propyl esters & $0.21 \pm 0.14^{\mathrm{a}}$ & $\mathrm{ND}^{\mathrm{b}}$ & $0.08 \pm 0.01^{\mathrm{a}}$ \\
\hline butyl esters & $\mathrm{ND}^{\mathrm{a}}$ & $\mathrm{ND}^{\mathrm{a}}$ & $0.03 \pm 0.00^{\mathrm{b}}$ \\
\hline higher alkyl esters & $\mathrm{ND}^{\mathrm{a}}$ & $\mathrm{ND}^{\mathrm{a}}$ & $\mathrm{ND}^{\mathrm{a}}$ \\
\hline branched alkyl esters & $1.82 \pm 0.85^{\mathrm{a}}$ & $0.06 \pm 0.01^{b}$ & $0.17 \pm 0.03^{b}$ \\
\hline Total esters & $4.46 \pm 2.27 \mathrm{a}$ & $0.17 \pm 0.03^{b}$ & $0.52 \pm 0.01^{b}$ \\
\hline alkanes & $0.72 \pm 0.42^{\mathrm{a}}$ & $0.43 \pm 0.28^{\mathrm{a}}$ & $0.46 \pm 0.69^{\mathrm{a}}$ \\
\hline aromatic hydrocarbons & $0.53 \pm 0.31^{\mathrm{a}}$ & $0.01 \pm 0.00^{\mathrm{b}}$ & $0.22 \pm 0.12^{b}$ \\
\hline unsaturated hydrocarbons & $16.27 \pm 15.38^{\mathrm{a}}$ & $0.01 \pm 0.00^{\mathrm{b}}$ & $0.01 \pm 0.00^{\mathrm{b}}$ \\
\hline Total hydrocarbons & $17.52 \pm 15.73^{a}$ & $0.44 \pm 0.28^{b}$ & $0.69 \pm 0.62^{b}$ \\
\hline sulphur compounds & $\mathrm{ND}^{\mathrm{a}}$ & $0.15 \pm 0.15^{\mathrm{b}}$ & $0.02 \pm 0.01^{b}$ \\
\hline terpenes & $2.02 \pm 0.84^{\mathrm{a}}$ & $0.08 \pm 0.01^{b}$ & $0.11 \pm 0.01^{\mathrm{b}}$ \\
\hline
\end{tabular}

a Method using P\&T-MWH. ${ }^{\mathrm{b}}$ Methods using P\&T-RH. ND: not detected.

a-c Means within rows without a common superscript are significantly different $(P \leq 0.05)$. 
Table VII. Percentage (means \pm SD; $n=4$ ) of the volatile compounds found in La Serena cheese using different DHS methods.

\begin{tabular}{|c|c|c|c|}
\hline & Method $A^{\text {a }}$ & Method $B^{b}$ & Method $C^{b}$ \\
\hline ethanoic acid & $\mathrm{ND}^{\mathrm{a}}$ & $0.56 \pm 0.29^{b}$ & $\mathrm{ND}^{\mathrm{a}}$ \\
\hline$n$-butanoic acid & $5.55 \pm 1.60^{\mathrm{a}}$ & $0.73 \pm 0.37 \mathrm{~b}$ & $0.01 \pm 0.01^{b}$ \\
\hline$n$-acids & $\mathrm{ND}^{\mathrm{a}}$ & $0.08 \pm 0.04^{b}$ & $\mathrm{ND}^{\mathrm{a}}$ \\
\hline branched-chain acids & $\mathrm{ND}^{\mathrm{a}}$ & $1.05 \pm 0.52^{b}$ & $\mathrm{ND}^{\mathrm{a}}$ \\
\hline Total acids & $5.55 \pm 1.60^{\mathrm{a}}$ & $2.41 \pm 1.18^{b}$ & $0.01 \pm 0.01 \mathrm{c}$ \\
\hline ethanol & $\mathrm{ND}^{\mathrm{a}}$ & $67.05 \pm 12.28^{b}$ & $51.40 \pm 1.82^{\mathrm{c}}$ \\
\hline 2-butanol & $\mathrm{ND}^{\mathrm{a}}$ & $5.49 \pm 3.52^{\mathrm{b}}$ & $3.86 \pm 3.12^{\mathrm{b}}$ \\
\hline primary alcohols & $7.40 \pm 2.52^{\mathrm{ab}}$ & $5.78 \pm 1.61^{\mathrm{a}}$ & $10.00 \pm 0.68^{b}$ \\
\hline secondary alcohols & $1.30 \pm 0.23^{\mathrm{a}}$ & $1.62 \pm 0.24^{\mathrm{a}}$ & $1.26 \pm 0.67^{\mathrm{a}}$ \\
\hline branched-chain alcohols & $6.36 \pm 4.77 \mathrm{a}$ & $4.56 \pm 1.31^{\mathrm{a}}$ & $18.03 \pm 1.03^{b}$ \\
\hline oxy ether alcohols & $\mathrm{ND}^{\mathrm{a}}$ & $0.09 \pm 0.02^{b}$ & $0.28 \pm 0.04^{\mathrm{c}}$ \\
\hline aromatic alcohols & $\mathrm{ND}^{\mathrm{a}}$ & $0.01 \pm 0.01^{b}$ & $0.02 \pm 0.01^{b}$ \\
\hline Total alcohols & $15.06 \pm 3.88^{a}$ & $84.58 \pm 6.44^{b}$ & $84.81 \pm 3.05^{b}$ \\
\hline$n$-aldehydes & $58.39 \pm 12.14^{\mathrm{a}}$ & $0.35 \pm 0.09^{b}$ & $0.52 \pm 0.11^{b}$ \\
\hline branched-chain aldehydes & $\mathrm{ND}^{\mathrm{a}}$ & $0.21 \pm 0.13^{b}$ & $0.54 \pm 0.13^{c}$ \\
\hline aromatic aldehydes & $\mathrm{ND}^{\mathrm{a}}$ & $\mathrm{ND}^{\mathrm{a}}$ & $\mathrm{ND}^{\mathrm{a}}$ \\
\hline Total aldehydes & $58.39 \pm 12.14^{a}$ & $0.56 \pm 0.06^{b}$ & $1.06 \pm 0.24^{b}$ \\
\hline methyl ketones & $3.22 \pm 2.08^{\mathrm{a}}$ & $7.96 \pm 5.11^{\mathrm{a}}$ & $1.71 \pm 1.68^{\mathrm{a}}$ \\
\hline propyl ketones & $\mathrm{ND}^{\mathrm{a}}$ & $\mathrm{ND}^{\mathrm{a}}$ & $\mathrm{ND}^{\mathrm{a}}$ \\
\hline aromatic ketones & $1.29 \pm 0.90^{\mathrm{a}}$ & $\mathrm{ND}^{\mathrm{b}}$ & $0.03 \pm 0.00^{c}$ \\
\hline diketones & $\mathrm{ND}^{\mathrm{a}}$ & $0.18 \pm 0.13^{b}$ & $0.61 \pm 0.19^{c}$ \\
\hline Total ketones & $4.51 \pm 2.86^{\mathrm{a}}$ & $8.13 \pm 5.22^{\mathrm{a}}$ & $2.35 \pm 1.86^{\mathrm{a}}$ \\
\hline methyl esters & $\mathrm{ND}^{\mathrm{a}}$ & $\mathrm{ND}^{\mathrm{a}}$ & $<0.01^{\mathrm{b}}$ \\
\hline ethyl esters & $13.34 \pm 9.71^{\mathrm{a}}$ & $2.94 \pm 0.50^{\mathrm{a}}$ & $10.90 \pm 4.70^{\mathrm{a}}$ \\
\hline propyl esters & $\mathrm{ND}^{\mathrm{a}}$ & $\mathrm{ND}^{\mathrm{a}}$ & $0.03 \pm 0.02^{b}$ \\
\hline butyl esters & $\mathrm{ND}^{\mathrm{a}}$ & $\mathrm{ND}^{\mathrm{a}}$ & $0.04 \pm 0.04^{b}$ \\
\hline higher alkyl esters & $\mathrm{ND}^{\mathrm{a}}$ & $0.01 \pm 0.00^{\mathrm{b}}$ & $\mathrm{ND}^{\mathrm{a}}$ \\
\hline branched alkyl esters & $0.87 \pm 0.80^{\mathrm{a}}$ & $0.11 \pm 0.03^{\mathrm{a}}$ & $0.40 \pm 0.16^{\mathrm{a}}$ \\
\hline Total esters & $14.20 \pm 10.17^{a}$ & $3.05 \pm 0.53^{a}$ & $11.38 \pm 4.87^{a}$ \\
\hline alkanes & $1.92 \pm 1.07 \mathrm{a}$ & $1.18 \pm 0.24^{\mathrm{ab}}$ & $0.20 \pm 0.07 b$ \\
\hline aromatic hydrocarbons & $0.37 \pm 0.19^{\mathrm{a}}$ & $\mathrm{ND}^{\mathrm{b}}$ & $0.09 \pm 0.04^{c}$ \\
\hline unsaturated hydrocarbons & $\mathrm{ND}^{\mathrm{a}}$ & $0.01 \pm 0.01^{\mathrm{b}}$ & $0.02 \pm 0.00^{\mathrm{b}}$ \\
\hline Total hydrocarbons & $2.29 \pm 1.20^{\mathrm{a}}$ & $1.19 \pm 0.24^{\mathrm{ab}}$ & $0.31 \pm 0.10^{b}$ \\
\hline sulphur compounds & $\mathrm{ND}^{\mathrm{a}}$ & $0.07 \pm 0.01^{\mathrm{b}}$ & $0.04 \pm 0.04^{b}$ \\
\hline terpenes & $\mathrm{ND}^{\mathrm{a}}$ & $\mathrm{ND}^{\mathrm{a}}$ & $0.04 \pm 0.01^{b}$ \\
\hline
\end{tabular}

a Method using P\&T-MWH. ${ }^{\text {b }}$ Methods using P\&T-RH. ND: not detected.

a-c Means within rows without a common superscript are significantly different $(P \leq 0.05)$. 
three methods were rather similar (Tabs. IIIVII).

The above mentioned results were partially in accordance with another study in which P\&T-RH was compared with P\&TMWH for the analysis of the volatile compounds of Swiss Emmental cheese [5]. The authors concluded that P\&T-RH was the most efficient for the determination of alcohols, carbonyl compounds, esters, hydrocarbons and sulphur compounds.

Considerable differences were also recorded in the percentages of the volatile compounds found in the PDO cheeses between the methods $\boldsymbol{B}$ and $\boldsymbol{C}$ using P\&TRH under different analytical conditions. A larger purge volume, higher temperatures, and higher proportion of $\mathrm{Na}_{2} \mathrm{SO}_{4}$ added to the cheese sample were used in method $C$ to carry out the DHS of cheese volatiles, compared with method $\boldsymbol{B}$. In general, larger percentages of acids and ketones were found by method $\boldsymbol{B}$ compared with method $\boldsymbol{C}$ in all the PDO cheese varieties, whereas the contrary occurred with the percentages of esters (Tabs. III-VII). Non-significant $(P>0.05)$ differences were found between the two methods for the percentages of alcohols in Zamorano, Manchego and La Serena cheeses, but larger percentages of alcohols, particularly 2-butanol, were found by method $\boldsymbol{C}$ compared with method $\boldsymbol{B}$ in Idiazabal and Roncal cheeses. Non-significant $(P>0.05)$ differences were also recorded for the percentages of other volatile compounds such as aldehydes, hydrocarbons and sulphur compounds in all of the PDO cheese varieties (Tables III-VII). Similar results were obtained by other authors in the analysis of the volatile compounds of Manchego, Roncal, Zamorano and La Serena cheeses by DHS using P\&T-RH [7, 15, 16, 25].

The coefficients of variation for the percentages of the volatile compounds found in all of the PDO cheese varieties by each of the three methods were, in general, higher than $10 \%$. However, the methods $\boldsymbol{B}$ and $\boldsymbol{C}$ which used automatic P\&T-RH showed lower coefficients of variation for most chemical families than those of the method $\boldsymbol{A}$, which used semi-automatic P\&T-MWH. The mean value for the coefficients of variation for all the chemical

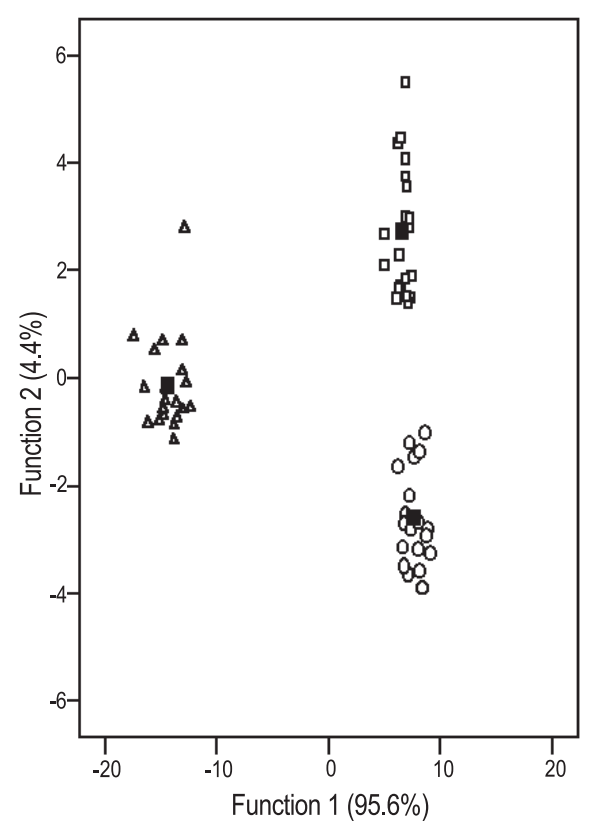

Figure 1. Cheese sample distribution according to DHS method used for volatile compound analysis in the two-dimensional coordinate system defined by canonical discriminant functions. (triangle): method $\boldsymbol{A}$ using P\&T-MWH; (circle): method $\boldsymbol{B}$ using P\&T-RH; (square): method $C$ using P\&T-RH.

families obtained by method $\boldsymbol{A}$ was $45.2 \%$, whereas 26.5 and $21.8 \%$ were the mean values obtained by methods $\boldsymbol{B}$ and $\boldsymbol{C}$, respectively. These results confirmed better repeatability for the DHS methods which used automatic P\&T-RH than for the DHS method which used semi-automatic P\&TMWH [23].

Stepwise discriminant analysis was applied to classify the cheese samples according to the DHS method used. The samples of the five PDO cheeses were correctly classified according to the method. Function 1, which accounted for $95.6 \%$ of variance, distinguished between method $\boldsymbol{A}$ and methods $\boldsymbol{B}$ and $\boldsymbol{C}$, whereas function 2, which accounted for only $4.4 \%$ of variance, distinguished between method $\boldsymbol{B}$ and method $\boldsymbol{C}$ (Fig. 1). Volatile compounds 

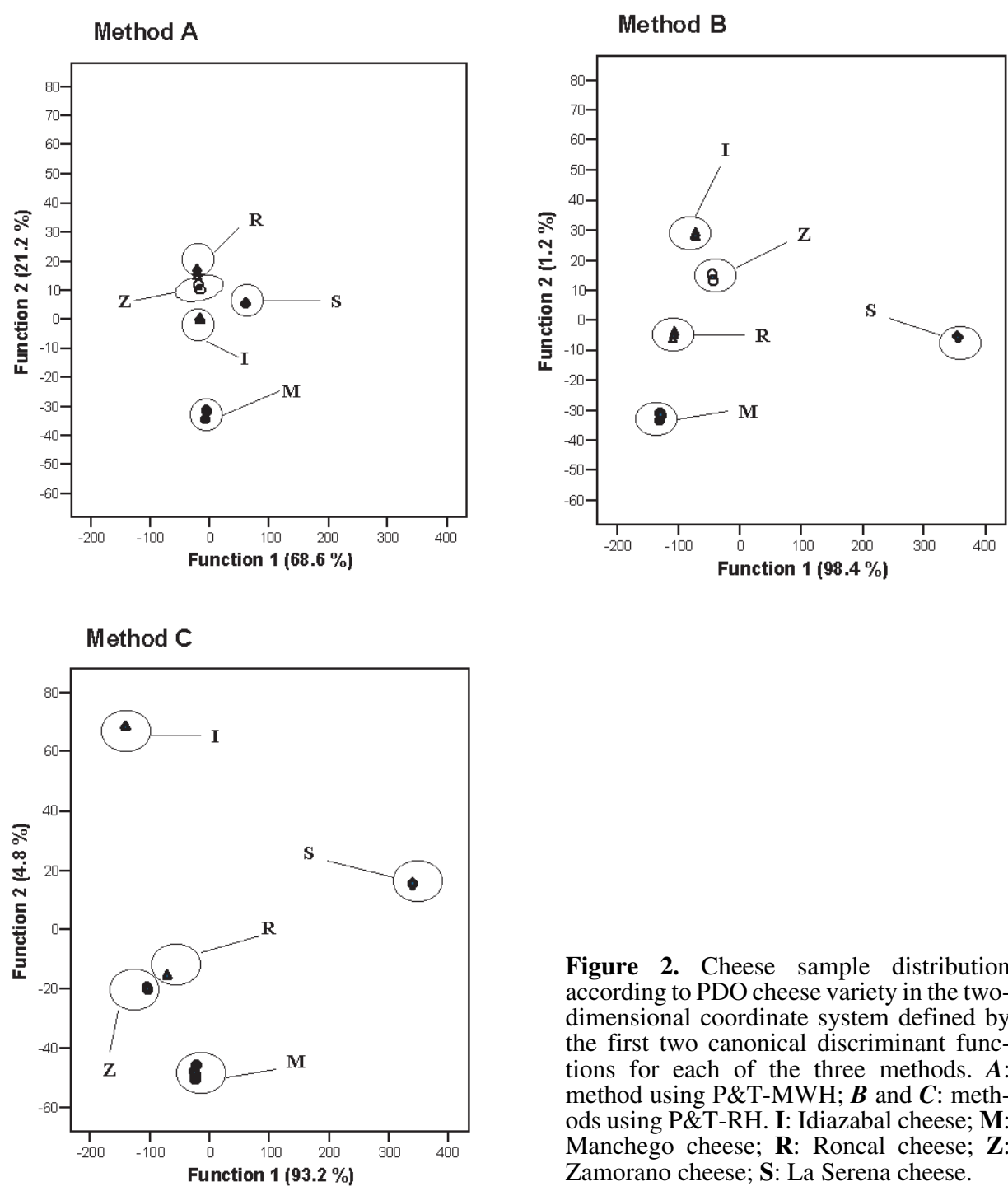

Figure 2. Cheese sample distribution according to PDO cheese variety in the twodimensional coordinate system defined by the first two canonical discriminant functions for each of the three methods. $\boldsymbol{A}$ : method using P\&T-MWH; $\boldsymbol{B}$ and $\boldsymbol{C}$ : methods using P\&T-RH. I: Idiazabal cheese; $\mathbf{M}$ : Manchego cheese; R: Roncal cheese; Z: Zamorano cheese; $\mathbf{S}$ : La Serena cheese.

such as sulphur compounds, total and secondary alcohols, methyl esters, $n$-butanoic acid and unsaturated hydrocarbons were closely correlated with function 1 , whereas straight, aromatic and total aldehydes, propyl esters, methyl ketones and aromatic hydrocarbons were closely correlated with function 2. These results showed clearly that, in this particular matter (volatile composition research), the results obtained by

different methods must be carefully compared, even if the same cheese piece is analysed by different laboratories using similar DHS systems.

Stepwise discriminant analyses were also applied separately to classify the PDO cheese varieties according to the volatile composition obtained by each of the three methods. As observed in Figure 2, the samples were correctly classified according to 
Table VIII. Discriminant variables included in the stepwise discriminant analyses applied to classify the PDO cheese varieties by different DHS methods.

\begin{tabular}{|c|c|c|}
\hline Method $\boldsymbol{A}^{\mathrm{a}}$ & Method $\boldsymbol{B}^{\mathrm{b}}$ & Method $\boldsymbol{C}^{\mathrm{b}}$ \\
\hline secondary alcohols & secondary alcohols & secondary alcohols \\
\hline ethyl esters & ethyl esters & propyl esters \\
\hline$n$-aldehydes & branched-chain alcohols & branched-chain alcohols \\
\hline$n$-acids & aromatic alcohols & aromatic alcohols \\
\hline ethanoic acid & ethanol & ethanol \\
\hline total acids & terpenes & oxy ether alcohols \\
\hline propyl ketones & branched alkyl esters & aromatic aldehydes \\
\hline \multirow[t]{3}{*}{ aromatic ketones } & higher alkyl esters & total ketones \\
\hline & aromatic hydrocarbons & branched-chain acids \\
\hline & & unsaturated hydrocarbons \\
\hline
\end{tabular}

a Method using P\&T-MWH. ${ }^{b}$ Methods using P\&T-RH.

the cheese PDO variety in each of the three methods. Function 1, accounting for 68.6, 98.4 and $93.2 \%$ of variance, respectively, for methods $\boldsymbol{A}, \boldsymbol{B}$ and $\boldsymbol{C}$, distinguished between La Serena cheese and the other four PDO cheese varieties. These results pointed out that the largest differences were recorded between the soft cheese variety made with vegetable rennet and the other semi-hard cheese varieties made with animal rennet. Function 2, accounting for 21.2, 1.2 and $4.8 \%$ of variance, respectively, for methods $\boldsymbol{A}, \boldsymbol{B}$ and $\boldsymbol{C}$, classified correctly the four semi-hard cheese varieties (Fig. 2). Although the three methods were capable of distinguishing between the volatile composition of the five PDO cheese varieties, the distances from each other for group centroids of each type of cheese were larger for methods $\boldsymbol{B}$ and $\boldsymbol{C}$ using automatic P\&T-RH than for method $\boldsymbol{A}$ using semi-automatic P\&T-MWH (Fig. 2). Table VIII shows the discriminant variables used to classify the PDO cheeses in each of the three methods. In general, different volatile compounds were used as discriminant variables for each of the three methods. It was remarkable that the secondary alcohols group was the only discriminant variable common to the three methods, and that different alcohol compounds were common to the methods which used automatic P\&T-RH (Tab. VIII). For method $\boldsymbol{A}$, volatile compounds such as $n$-acids, total acids, ethanoic acid, $n$-aldehydes or ethyl esters, whose percentages showed large differences among the PDO cheeses, were used as discriminant variables. For methods $\boldsymbol{B}$ and $\boldsymbol{C}$, volatile compounds such as ethanol, branched-chain alcohols and total ketones, whose percentages showed large differences among the PDO cheeses, were used as discriminant variables.

In summary, the results pointed out differences in the extraction of volatile compounds from the cheese samples according to the different DHS system used, that were semi-automatic P\&T-MWH and automatic P\&T-RH. The nature of the trap adsorbent, i.e. graphitised carbon for P\&T-MWH and tenax for P\&T-RH, was determinant for the selectivity of the adsorption of cheese volatiles. Differences in the extraction of volatile compounds were also observed between laboratories which used the same DHS system under different analytical conditions, i.e. different automatic sampler model, sample preparation, and conditions for purge, concentration on trap, and thermal desorption.

For the comparison of different cheese varieties manufactured in the same period, the DHS methods used in this study are equally satisfactory as shown by the correct classification of the 4 samples from 2 batches of each cheese variety by the discriminant analyses. However, the results on 
volatile composition obtained by different methods must be carefully compared, even if the same cheese piece is analysed using similar DHS systems under different analytical conditions. The P\&T-MWH and P\&T-RH systems assayed in this work have advantages and disadvantages for the exhaustive characterisation of the aroma compounds of a particular cheese. For the largest lipolysed cheeses such as Idiazabal, Roncal and Zamorano, P\&T-MWH provides only a relatively poor aroma profile which is mainly composed of volatile acids, whereas P\&T-RH gives more information on other aroma compounds such as methyl ketones. For the lowest lipolysed cheeses such as Manchego and La Serena, P\&T-RH provides a mainly alcoholic profile, whereas P\&T-MWH gives more information on other aroma components such as volatile acids and aldehydes.

Acknowledgements: The authors thank the Regulatory Councils of the PDOs of "Idiazabal", "Roncal", "Queso de La Serena", "Queso Manchego" and "Queso Zamorano", and Quesería Balda, Quesería Larra, Quesería Artesanal Vicente Pastor, Finca La Prudenciana and Quesería El Jardín for providing the commercial cheese samples. This work was supported by grant SC98-096 from the National Institute of Agricultural and Food Research and Technology (Madrid, Spain). Y. Redondo acknowledges a pre-doctoral fellowship from the University of the Basque Country (Leioa, Spain). M. Puy Portillo prepared the French translation.

\section{REFERENCES}

[1] Almarcha M., Rovira J., Análisis de compuestos orgánicos volátiles (VOC) mediante atrapamiento en adsorbentes carbonáceos y desorción térmica mediante microondas guiadas, Técn. Lab. 164 (1991) 322-327.

[2] Ayad E.H.E., Awad S., El Attar A., de Jong C., El-Soda M., Characterisation of Egyptian Ras cheese. 2. Flavour formation, Food Chem. 86 (2004) 553-561.

[3] Barroso M., Barron L.J.R., Optimization of the analytical conditions dealing with the volatile fraction of cheese using dynamic headspace and trap desorption by means of microwave energy, in: Sontag G., Pfannhauser W. (Eds.), Current Status and Future Trends, vol. 1,
Proceedings of EURO FOOD CHEM VIII, Wageningen Press, Wageningen, Germany, 1995, pp. 18-25.

[4] Boscaini E., van Ruth S., Biasioli F., Gasperi F., Maerk T.D., Gas chromatography-olfactometry (GC-O) and proton transfer reaction-mass spectrometry (PTR-MS) analysis of the flavour profile of Grana Padano, Parmiggiano Reggiano, and Grana Trentino cheeses, J. Agric. Food Chem. 51 (2003) 1782-1790.

[5] Bosset J.O., Gauch R., Mariaca R., Klein B., Comparison of various sample treatments for the analysis of volatile compounds by GC-MS: Application to the Swiss Emmental cheese, Mitt. Gebiete Lebensm. Hyg. 86 (1995) 672-698.

[6] Carbonell M., Núñez M., Fernández-García E., Evolution of the volatile components of ewe raw milk La Serena cheese during ripening. Correlation with flavour characteristics, Lait 82 (2002) 683-698.

[7] Carbonell M., Núñez M., Fernández-García E., Seasonal variation of volatile compounds in ewe raw milk La Serena cheese, Lait 82 (2002) 699-711.

[8] Careri M., Manini P., Spagnoli S., Barbieri G., Bolozoni L., Simultaneous-distillationextraction and dynamic headspace methods in the gas chromatographic analysis of Parmesan cheese volatiles, Chromatographia 38 (1994) 386-394.

[9] Chávarri F., Bustamante M.A., Santisteban A., Virto M., Barron L.J.R., de Renobales M., Changes in free fatty acids during ripening of Idiazabal cheese manufactured at different times of the year, J. Dairy Sci. 82 (1999) 885-890.

[10] Chávarri F., Bustamante M.A., Santisteban A., Virto M., Albisu M., Barron L.J.R., de Renobales M., Effect of milk pasteurization on lipolysis during ripening of Idiazabal cheese manufactured at different times of the year, Lait 80 (2000) 433-444.

[11] Curioni P.M.G., Bosset J.O., Key odorants in various cheese types as determined by gas chromatography-olfactometry, Int. Dairy J. 12 (2002) 959-984.

[12] Di Cagno R., Banks J., Sheehan L., Fox P.F., Brechany E.Y., Corsetti A., Gobbetti M., Comparison of the microbiological, compositional, biochemical, volatile profile and sensory characteristics of three Italian PDO ewes' milk cheeses, Int. Dairy J. 13 (2003) 961-972.

[13] Dirink P., De Winne A., Flavour characterisation and classification of cheeses by gas chromatographic-mass spectrometric profiling, J. Chromatogr. A 847 (1999) 203-208. 
[14] Fernández-García E., Use of headspace sampling in the quantitative analysis of artisanal Spanish cheese aroma, J. Agric. Food Chem. 44 (1996) 1833-1839.

[15] Fernández-García E., Carbonell M., Núñez M., Volatile fraction and sensory characteristics of Manchego cheese. 1. Comparison of raw and pasteurized milk cheese, J. Dairy Res. 69 (2002) 579-593.

[16] Fernández-García E., Carbonell M., Gaya P., Núñez M., Evolution of the volatile components of ewes raw milk Zamorano cheese. Seasonal variation, Int. Dairy J. 14 (2004) 701-711.

[17] Fernández-García E., Gaya P., Medina M., Núñez M., Evolution of the volatile components of raw ewes' milk Castellano cheese: seasonal variation, Int. Dairy J. 14 (2004) 39-46.

[18] Ferraza R.E., Fresno J.M., Riberiro J.I., Tornadejo M.E., Mansur Furtado M., Changes in the microbial flora of Zamorano cheese (P.D.O.) by accelerated ripening process, Food Res. Int. 37 (2004) 149-155.

[19] Freitas A.C., Macedo A.C., Malcata F.X., Review: technological and organoleptic issues pertaining to cheeses with denomination of origin manufactured in the Iberian Peninsula from ovine and caprine milks, Food Sci. Technol. Int. 6 (2000) 351-370.

[20] Gallois A., Langlois F., New results in the volatile odorous compounds of French cheeses, Lait 70 (1990) 89-106.

[21] Gómez-Ruiz J.A., Ballesteros C., González Viñas M.A., Cabezas L., Martínez-Castro I., Relationships between volatile compounds and odour in Manchego cheese: comparison between artisanal and industrial cheeses at different ripening times, Lait 82 (2002) 613628.

[22] Hair J.F., Anderson R.E., Tatham R.L., Black W.C., Multivariate data analysis, 5th edn., Prentice-Hall, London, UK, 1998.

[23] Imhof R., Bosset J.O., Comparison of two systems for sample preparation and injection by dynamic headspace GC analysis, J. High Resol. Chromatogr. Comm. 14 (1991) 621625.

[24] Irigoyen A., Izco J.M., Ibáñez F.C., Torre P., Influence of rennet milk-clotting activity on the proteolytic and sensory characteristics of an ovine cheese, Food Chem. 72 (2001) 137-144.

[25] Izco J.M., Torre P., Characterisation of volatile flavour compounds in Roncal cheese extracted by the "purge and trap" method and analysed by GC-MS, Food Chem. 70 (2000) 409-417.
[26] Larráyoz P., Carbonell M., Ibáñez F.C., Torre P., Barcina Y., Optimization of indirect parameters which affect the extractability of volatile aroma compounds from Idiazabal cheese using analytical supercritical fluid extraction (SFE), Food Chem. 64 (1999) 123-127.

[27] Larráyoz P., Addis M., Gauch R., Bosset J.O., Comparison of dynamic headspace and simultaneous distillation extraction techniques used for the analysis of the volatile components in three European PDO ewe's milk cheeses, Int. Dairy J. 11 (2001) 911-926.

[28] Lawlor J.B., Delahunty C.M., Wilkinson M.G., Sheehan J., Relationships between the gross, non-volatile and volatile compositions and the sensory attributes of eight hardtype cheeses, Int. Dairy J. 12 (2002) 493-509.

[29] Lawlor J.B., Delahunty C.M., Sheehan J., Wilkinson M.G., Relationships between sensory attributes and the volatile compounds, non-volatile and gross compositional constituents of six blue-type cheeses, Int. Dairy J. 13 (2003) 481-494.

[30] Lecanu L., Ducruet V., Jouquand C., Gratadoux J.J., Feigenbaum A., Optimization of headspace soli-phase microextraction (SPME) for the odor analysis of surface-ripened cheese, J. Agric. Food Chem. 50 (2002) 3810-3817.

[31] Lee J.H., Diono R., Kim G.Y., Min D., Optimization of solid phase microextraction analysis for the headspace volatile compounds of Parmesan cheese, J. Agric. Food Chem. 51 (2003) 1136-1140.

[32] Mariaca R., Bosset J.O., Instrumental analysis of volatile (flavour) compounds in milk and dairy products, Lait 77 (1997) 13-40.

[33] Ministerio de Agricultura, Pesca y Alimentación, Reglamento de la Denominación de Origen Roncal y de su Consejo Regulador, Bol. O. Estado 63 (1991) 8489.

[34] Ministerio de Agricultura, Pesca y Alimentación, Reglamento de la Denominación de Origen Idiazabal y de su Consejo Regulador, Bol. O. Estado 289 (1993) 34591.

[35] Ministerio de Agricultura, Pesca y Alimentación, Reglamento de la Denominación de Origen Queso Zamorano y su Consejo Regulador, Bol. O. Estado 120 (1993) 115311.

[36] Ministerio de Agricultura, Pesca y Alimentación, Reglamento de la Denominación de Origen Queso de La Serena y su Consejo Regulador, Bol. O. Estado 100 (1993) 12501.

[37] Ministerio de Agricultura, Pesca y Alimentación, Reglamento de la Denominación de Origen Queso Manchego y de su Consejo Regulador, Bol. O. Estado 295 (1995) 35548. 
[38] Moio L., Piombino P., Addeo F., Odourimpact compounds of Gorgonzola cheese, $\mathrm{J}$. Dairy Res. 67 (2000) 273-285.

[39] Muñoz N., Ortigosa M., Torre P., Izco J.M., Free amino acids and volatile compounds in an ewe's milk cheese as affected by seasonal and cheese-making plant variations, Food Chem. 83 (2003) 329-338.

[40] Núñez A.J., González L.F., Janák J., Preconcentration of headspace volatiles for trace organic analysis by gas chromatography, J. Chromatogr. 300 (1984) 127-162.

[41] O’Riordan P.J., Delahunty C.M., Characterisation of commercial Cheddar cheese flavour. 2: study of Cheddar cheese discrimination by composition, volatile compounds and descriptive flavour assessment, Int. Dairy J. 13 (2003) 371-389.

[42] Ortigosa M., Torre P., Izco J.M., Effect of pasteurization of ewe's milk and use of a native starter culture on the volatile components and sensory characteristics of Roncal cheese, J. Dairy Sci. 84 (2001) 1320-1330.

[43] Pérez-Elortondo F.J., Aldámiz-Echebarria P., Albisu M., Barcina Y., Indigenous lactic acid and bacteria in Idiazabal ewe's milk cheese, Int. Dairy J. 8 (1998) 725-732.

[44] Póveda J., Pérez-Coello M.S., Cabezas L., Evolution of the free fatty acid fraction in Manchego cheese during ripening, Milchwissenschaft 54 (1999) 685-687.

[45] Rektorik J., Head-space and desorption by microwaves, Riv. Ital. EPPOS 4 (1993) 165-173.

[46] Roa I., López M.B., Mendiola F.J., Residual clotting activity and ripening properties of vegetable rennet from Cynara cardunculus in La Serena cheese, Food Res. Int. 32 (1999) 413-419.

[47] Stefanon B., Procida G., Effects of including silage in the diet on voaltile compound profiles in Montasio cheese and their modification during ripening, J. Dairy Res. 71 (2004) $58-65$.

[48] Thierry A., Maillard M.B., Hervé C., Richoux R., Lortal S., Varied volatile compounds are produced by Propionibacterium freudenreichii in Emmental cheese, Food Chem. 87 (2004) 439-446.

[49] Valero E., Villaseñor M.J., Sanz J., MartínezCastro I., Comparison of two methods based on dynamic headspace for GC-MS analysis of volatile components of cheeses, Chromatographia 52 (2000) 340-344.

[50] Valero E., Sanz J., Martínez-Castro I., Direct thermal desorption in the analysis of cheese volatiles by gas chromatography and gas chromatography-mass spectrometry: comparison with simultaneous distillationextraction and dynamic headspace, J. Chromatogr. Sci. 39 (2001) 222-228.

[51] Villaseñor M.J., Valero E., Sanz J., MartínezCastro I., Analysis of volatile compounds of Manchego cheese by dynamic headspace followed by automatic thermal desorption-GCMS, Milchwissenschaft 55 (2000) 378-382.

[52] Wilkes J.G., Conte E.D., Kim Y., Holcomb M., Sutherland J.B., Miller D.W., Sample preparation for the analysis of flavours and off-flavours in foods, J. Chromatogr. A 880 (2000) 3-33. 